\title{
ZHANG'S CONJECTURE AND THE EFFECTIVE BOGOMOLOV CONJECTURE OVER FUNCTION FIELDS
}

\author{
ZUBEYIR CINKIR
}

\begin{abstract}
We prove the Effective Bogomolov Conjecture, and so the Bogomolov Conjecture, over a function field of characteristic 0 by proving Zhang's Conjecture about certain invariants of metrized graphs. In the function field case, these conjectures were previously known to be true only for curves of genus at most 4 and a few other special cases. We also either verify or improve the previous results. We relate the invariants involved in Zhang's Conjecture to the tau constant of metrized graphs. Then we use and extend our previous results on the tau constant. By proving another Conjecture of Zhang, we obtain a new proof of the slope inequality for Faltings heights on moduli space of curves.
\end{abstract}

\section{INTRODUCTION}

In this paper, we study various invariants associated to a given metrized graph and polarized metrized graph. We derive formulas relating the invariants studied in the papers [Zh2 and [Fa] in terms of the tau constant of metrized graphs. This enables us to use the tools developed ( $[\mathrm{CR}],[\mathrm{BR},[\mathrm{BF},[\mathrm{C} 1],[\mathrm{C} 2], \mathrm{C} 3]$, and [C4]) to study the tau constant. We extend our previous results on the tau constant ([C1], [C2] and [C3]), prove S. Zhang's Conjecture [Zh2, 4.1.2], and prove stronger version of X. Faber's Conjecture [Fa, 1.3]. The consequences of these conjectures include the following applications in number theory and algebraic geometry:

(i) We prove the effective Bogomolov's Conjecture over function fields of characteristic 0 . If a conjecture (see the articles [Zh2, 1.4.1] and [GS]) due to Grothendieck and GilletSoule is true, our results extend to the function field of positive characteristic case, and have implications to number field case. The Bogomolov Conjecture over function fields were previously known only in some special cases, which will be discussed briefly in the next section.

(ii) We give a new proof of a slope inequality for Faltings heights on moduli space of curves by proving another Conjecture of Zhang [Zh2, Conjecture 1.4.5]. This slope inequality was first proved by A. Moriwaki in the article [AM4, Theorem D at page 3] in the characteristic 0 case and in the article AM2, Theorem 4.1] for arbitrary characteristic. Our method depends only on calculations involving invariants of metrized graphs, and makes it possible to obtain stronger versions of the slope inequality in certain special cases.

Throughout the paper, we use the interpretation of metrized graphs as resistive electric circuits and related electrical properties such as circuit reductions. Whenever it is needed, we consider metrized graphs only with their combinatorial graph structure. Our previous

Key words and phrases. Zhang's Conjecture, Bogomolov Conjecture, Effective Bogomolov Conjecture, Tau Constant, Metrized Graphs, slope inequality, discrete Laplacian matrix.

I would like to thank R. Rumely and M. Baker for their continued support and guidance. I would like to thank X. Faber for letting me know about Zhang's Conjecture and for useful correspondence during the preparation of this paper. 
results on the tau constant ([C1], [C2], and [C3]) use the properties of a continuous Laplacian operator on a metrized graph, which was defined by M. Baker and R. Rumely [BR] and studied from the perspective of harmonic analysis. We will give a brief description of metrized graphs, and recall results from electric circuit theory and combinatorial graph theory with short explanations. Interested readers should consult the references cited in the related sections.

\section{The Bogomolov Conjecture, the slope inequality and main Results}

We first recall some definitions. Let $X$ be a smooth projective surface over a field $k$, and let $Y$ be a smooth projective curve over $k$. A fibration $f: X \longrightarrow Y$ over $Y$ is called "isotrivial", if all smooth fibers are isomorphic to a fixed curve.

Let $k$ be a field. Let $X$ be a smooth projective surface over $k$, and let $Y$ be a smooth projective curve over $k$. Let $f: X \rightarrow Y$ be a semi-stable fibration such that the generic fiber of $f$ is smooth and of genus $\bar{g} \geq 2$. Let $K$ be the function field of $Y$, with algebraic closure $\bar{K}$, and let $C$ be the generic fiber of $f$. The Néron-Tate height pairing on the Jacobian variety $\operatorname{Jac}(C)(\bar{K})=P i c^{0}(C)(\bar{K})$ of the curve $C / K$ induces a seminorm $\|\cdot\|_{N T}$. For $\frac{\omega_{C}}{2 \bar{g}-2} \in P i c^{1}(C)(\bar{K})$, we have a canonical inclusion $j: C(\bar{K}) \longrightarrow \operatorname{Jac}(C)(\bar{K})$ defined by $j(x)=(2 g-2) x-\omega_{C}$.

If we define $B_{C}(P ; r)=\left\{x \in C(\bar{K}):\|j(x)-P\|_{N T} \leq r\right\}$, where $P \in \operatorname{Pic}^{0}(C)(\bar{K})$ and $r \geq 0$, and if we set

$$
r_{C}(P)= \begin{cases}-\infty & \text { if } \#\left(B_{C}(P ; 0)\right)=\infty \\ \sup \left\{r \geq 0 \mid \#\left(B_{C}(P ; r)\right)<\infty\right\} & \text { otherwise. }\end{cases}
$$

then Bogomolov's conjecture can be stated as follows:

Conjecture 2.1. AM2](Bogomolov Conjecture) If $f$ is non-isotrivial, then $r_{C}(P)>0$ for all $P$.

Conjecture 2.2. [KY1](Effective Bogomolov Conjecture) If $f$ is non-isotrivial, then there exists an "effectively calculable" positive number $r_{0}$ such that

$$
\inf _{P \in P i c^{0}(C)(\bar{K})} r_{C}(P) \geq r_{0} .
$$

We will now describe how metrized graphs can be related to above conjectures by following the articles [AM2] and [Zh1.

For the semistable fibration $f: X \longrightarrow Y$, let $C V(f)=\left\{y_{1}, y_{2}, \cdots, y_{s}\right\}$ be the set of critical values of $f$, where $s$ is the number of singular fibers. That is, $y \in C V(f)$ iff $f^{-1}(y)$ is singular. For any $y_{i} \in C V(f)$, let $\Gamma_{y_{i}}$ be the dual graph of the fiber $C_{y_{i}}:=f^{-1}\left(y_{i}\right)$, for each $1 \leq i \leq s$. The metrized graph $\Gamma_{y_{i}}$ is obtained as follows. The set of vertices $V_{y_{i}}$ of $\Gamma_{y_{i}}$ is indexed by irreducible components of the fiber $f^{-1}\left(y_{i}\right)$ and the singularities of $f^{-1}\left(y_{i}\right)$ correspond to edges of length 1 . Let $I\left(C_{y_{i}}\right):=\left\{C_{1, y_{i}}, C_{2, y_{i}}, \cdots, C_{v_{i}, y_{i}}\right\}$ be the set of irreducible components of the fiber $C_{y_{i}}$, where $v_{i}$ is the number of irreducible components in $C_{y_{i}}$. Then the irreducible curve $C_{j, y_{i}}$ corresponds to the vertex $p_{j} \in V_{y_{i}}$ for each $1 \leq j \leq v_{i}$ (see Figure 5 and Figure 1). Let $\delta_{y_{i}}$ be the number of singularities in $C_{y_{i}}$. By our construction, $\delta_{y_{i}}=\ell\left(\Gamma_{y_{i}}\right)$, the length of $\Gamma_{y_{i}}$, for each $1 \leq i \leq s$. Let $\delta:=\sum_{i=1}^{s} \delta_{y_{i}}$, the total number of singularities in the fibration. For any $p_{j} \in V_{y_{i}}$, let $\mathbf{q}\left(p_{j}\right):=g\left(C_{j, y_{i}}\right)$, where $g\left(C_{j, y_{i}}\right)$ is the arithmetic genus of $C_{j, y_{i}}$ (see $\oiint_{4}$ for the role of $\mathbf{q})$. Let $g(Y)$ be the genus of $Y$. 


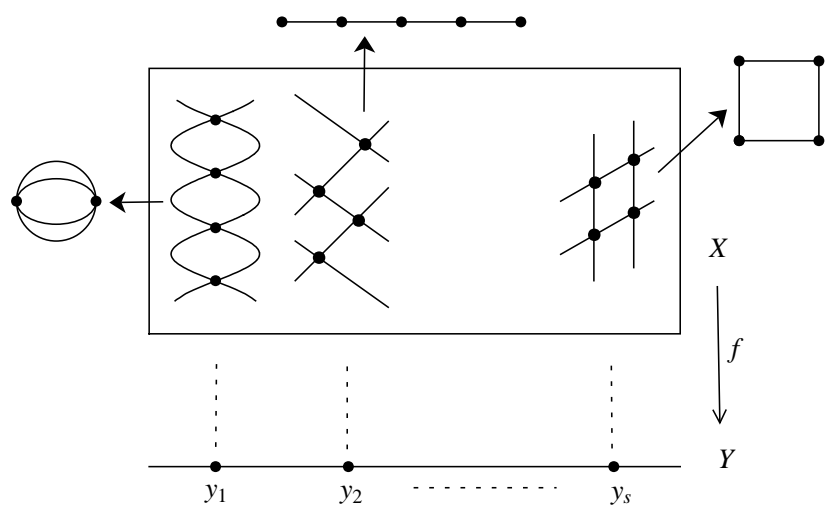

Figure 1. Singular fibers of $f: X \rightarrow Y$, and dual graphs of fibers.

We have $K_{X}, K_{Y}, \omega_{X}, \omega_{Y}$, and $\omega_{X / Y}$, which are the canonical divisor of $X$, the canonical divisor of $Y$, the dualizing sheaf of $X$, the dualizing sheaf of $Y$, and the relative dualizing sheaf, respectively. For the admissible dualizing sheaf $\omega_{a}$ [Zh1] (see also the article [AM1, pg. 3]), we have the following inequalities due to Zhang [Zh1]:

$$
\omega_{X / Y}^{2} \geq \omega_{a}^{2} \geq 0
$$

Zhang [Zh1] showed that $\omega_{a}^{2}>0$ is equivalent to the Bogomolov conjecture, i.e., Conjecture 2.1.

Theorem 2.3. AM3, Theorem 2.1] [Zh1, Theorem 5.6] If $\omega_{a}^{2}>0$, then

$$
i n f_{P \in P i c^{0}(C)(\bar{K})} r_{C}(P) \geq \sqrt{(g-1) \omega_{a}^{2}} \text {. }
$$

Let $\epsilon(\Gamma)$ and $\varphi(\Gamma)$ be the invariants of a dual graph $\Gamma$ defined in $\$ 4$ below. We have ([Zh2, Equation 1.2.1], [AM3, Equation 2.2], and [C1, Section 4.5])

$$
\omega_{a}^{2}=\omega_{X / Y}^{2}-\sum_{i=1}^{s} \epsilon\left(\Gamma_{y_{i}}\right)
$$

Zhang defined the canonical Gross-Schoen cycle $\Delta_{\xi}$ associated to $X$, and showed in the article [Zh2, Corollary 1.3.2] that

$$
\omega_{a}^{2}=\frac{2 \bar{g}-2}{2 \bar{g}+1}\left\langle\Delta_{\xi}, \Delta_{\xi}\right\rangle+\frac{2 \bar{g}-2}{2 \bar{g}+1} \sum_{i=1}^{s} \varphi\left(\Gamma_{y_{i}}\right) .
$$

Remark 2.4. Since the height of $\Delta_{\xi}$, namely $\left\langle\Delta_{\xi}, \Delta_{\xi}\right\rangle$, is non-negative whenever the characteristic of $k$ is 0 (as in Theorem 2.12 and Theorem 2.14), proving the positivity of $\varphi(\Gamma)$ for any polarized metrized graph (pm-graph in short) $\Gamma$ will be enough to prove Bogomolov Conjecture.

Remark 2.5. Whenever $f$ is smooth, we clearly have $\omega_{a}^{2}=\omega_{X / Y}^{2}$ by Equation (2), and that $\omega_{X / Y}^{2} \geq 12$ as Paršin $[\mathrm{P}]$ showed.

The Effective Bogomolov Conjecture (Conjecture 2.2) was known to be true for curves of genus less than 5 ([AM1, [AM2], [AM3], [AM4], KY1], [KY2], and [Fa]). Also, W. Gubler [G] showed that the Bogomolov Conjecture is true for $C$ if the Jacobian variety of $C$ has 
totally degenerate reduction over some point $y \in Y$. One can consult the article [Fa] to see the list of previously known lower bounds to $\omega_{a}^{2}$.

We will use the following notation for the singularities that are in the fibers of $f$ :

Let $y \in C V(f)$, and let $p \in f^{-1}(y)$ be a node. If the partial normalization of $f^{-1}(y)$ at $p$ is connected, we say that $p$ is of type 0 . If it is disconnected, then it has two components, in which case $p$ will be said to be of type $i$, where $i$ is the minimum of the arithmetic genera of the components. We denote the total number of nodes of type $i$ in the fiber $f^{-1}(y)$ by $\delta_{i}\left(\Gamma_{y}\right)$, and we set $\delta_{i}(X)=\sum_{j=1}^{s} \delta_{i}\left(\Gamma_{y_{j}}\right)$. With our earlier notation, we have $\delta_{y_{j}}=\sum_{i \geq 0} \delta_{i}\left(\Gamma_{y_{j}}\right)$ and $\delta=\sum_{i \geq 0} \delta_{i}(X)$.

Next we state Zhang's first conjecture which implies Conjecture 2.2, and so Conjecture 2.1.

Conjecture 2.6. [Zh2, Conjecture 1.4.2] For any $y \in C V(f)$, there is a positive continuous function $c(\bar{g})$ of $\bar{g} \geq 2$ such that the following inequality holds:

$$
\varphi\left(\Gamma_{y}\right) \geq c(\bar{g}) \delta_{0}\left(\Gamma_{y}\right)+\sum_{i \geq 1} \frac{2 i(\bar{g}-i)}{\bar{g}} \delta_{i}\left(\Gamma_{y}\right) .
$$

Previous results, due to Moriwaki and K. Yamaki when $\bar{g}$ is 2 or 3, on Conjecture 2.2 depend on a slope inequality which is the following lower bound for $\operatorname{deg} f_{*}\left(\omega_{X / Y}\right)$ :

$$
\operatorname{deg} f_{*}\left(\omega_{X / Y}\right) \geq \frac{\bar{g}}{8 \bar{g}+4} \delta_{0}(X)+\sum_{i \geq 1} \frac{i(\bar{g}-i)}{2 \bar{g}+1} \delta_{i}(X) .
$$

Note that the inequality (4) was proved by Moriwaki [AM4, Theorem D at page 3] in the characteristic 0 case. This slope inequality is actually closely related to the inequality given in the article [AM2, Theorem 4.1], which is slightly weaker but holds in any characteristic. Its proof and connection to Bogomolov Conjecture is through the following equation which is obtained by Noether's formula

$$
\operatorname{deg} f_{*}\left(\omega_{X / Y}\right)=\frac{1}{12}\left(\omega_{X / Y}^{2}+\sum_{i=1}^{s} \delta_{y_{i}}\right) .
$$

Let $\lambda(\Gamma)$ and $a(\Gamma)$ be the invariants of a dual graph $\Gamma$ defined in $\$ 4$ below. We have Zhang's second conjecture leading to a second proof of the slope inequality given in (44).

Conjecture 2.7. [Zh2, Conjecture 1.4.5] For any $y \in C V(f)$, the following inequality holds:

$$
\lambda\left(\Gamma_{y}\right) \geq \frac{\bar{g}}{8 \bar{g}+4} \delta_{0}\left(\Gamma_{y}\right)+\sum_{i \geq 1} \frac{i(\bar{g}-i)}{2 \bar{g}+1} \delta_{i}\left(\Gamma_{y}\right) .
$$

Zhang reduced and unified his first and second conjectures into the following conjecture:

Conjecture 2.8. [Zh2, Conjecture 4.1.2] Let $\Gamma$ be an irreducible polarized metrized graph of genus $\bar{g}$. Then the following two inequalities hold:

$$
\frac{\bar{g}-1}{\bar{g}+1}(\ell(\Gamma)-4 \bar{g} \cdot a(\Gamma)) \leq \epsilon(\Gamma) \leq 12 \bar{g} \cdot a(\Gamma)-(1+c(\bar{g})) \ell(\Gamma),
$$

where $c(\bar{g})$ is a positive number for each $\bar{g} \geq 2$.

Faber verified this conjecture for curves of genus less than 5. In the rest of this section, we will state our main results.

We prove that Conjecture 2.6 holds as follows: 
Theorem 2.9. Let $\Gamma$ be a pm-graph with genus $\bar{g}$. Then we have

$$
\varphi(\Gamma) \geq t(\bar{g}) \delta_{0}(\Gamma)+\sum_{i \geq 1} \frac{2 i(\bar{g}-i)}{\bar{g}} \delta_{i}(\Gamma)
$$

where $t(2)=\frac{1}{27}, t(3)=\frac{892-11 \sqrt{79}}{14580} \approx 0.054473927$, and $t(\bar{g})=\frac{(\bar{g}-1)^{2}}{2 \bar{g}(7 \bar{g}+5)}$ for $\bar{g} \geq 4$. In particular, $t(\bar{g}) \geq \frac{3}{88}$ for $\bar{g} \geq 4$.

Proof. The result follows from Theorem 5.21, Theorem 7.8 and the article [Zh2, Corollary 4.4.2].

We believe that the lower bounds in Theorem 2.9 can be improved when $\bar{g} \geq 3$. In the light of Proposition 5.7, which gives an exact formula for $\varphi(\Gamma)$ when $\Gamma$ is a complete graph, the lower bounds in Theorem 2.9 are not far from optimal. In \$5, we give better bounds to $\varphi(\Gamma)$ for certain classes of pm-graphs. Corollary 5.25 shows that $\varphi(\Gamma)$ can be very large for some pm-graphs.

We prove that Conjecture 2.7 holds as follows (with notation as in 93 ):

Theorem 2.10. Let $(\Gamma, \boldsymbol{q})$ be a pm-graph. Then we have

$$
\lambda(\Gamma) \geq \frac{\bar{g}}{8 \bar{g}+4} \delta_{0}(\Gamma)+\sum_{i \geq 1} \frac{i(\bar{g}-i)}{2 \bar{g}+1} \delta_{i}(\Gamma) .
$$

Proof. The result follows from Proposition 4.13 and the article [Zh2, Corollary 4.4.2].

For any given genus $\bar{g}$, we have examples showing that $\lambda(\Gamma)$ can be very close to the lower bound given in Theorem 2.10 or it can be linear in $\bar{g}$ (see Corollary 5.25 ). Therefore, the topology of the pm-graph $\Gamma$ plays important role in the value of $\lambda(\Gamma)$. The techniques we have developed can be used for numeric calculations of $\lambda(\Gamma)$ in general and symbolic calculations of $\lambda(\Gamma)$ in specific cases.

Theorem 2.11. Conjecture 2.8 holds with $c(\bar{g})=4 t(\bar{g})$ for $\bar{g} \geq 2$, where $t(\bar{g})$ is as in Theorem 2.9.

Proof. The result follows from Theorem 2.9, Theorem 2.10 and Lemma 4.10,

Theorem 2.12. Let char $(k)=0$. If $f$ is non-isotrivial, then we have inf $\operatorname{PAPic}^{0}(C)(\bar{K}) r_{C}(P) \geq$ $\sqrt{r_{0}}$, where $r_{0}$ can be taken as follows:

$$
r_{0}= \begin{cases}12(\bar{g}-1), & \text { if } f \text { is smooth } \\ \frac{2(\bar{g}-1)^{2}}{2 \bar{g}+1}\left(t(\bar{g}) \delta_{0}(\Gamma)+\sum_{i \geq 1} \frac{2 i(\bar{g}-i)}{\bar{g}} \delta_{i}(\Gamma)\right), & \text { otherwise }\end{cases}
$$

with $t(\bar{g})$ as in Theorem [2.9. Therefore, the Effective Bogomolov Conjecture holds.

Proof. If $f$ is smooth, then the result follows from Remark 2.5 and Theorem 2.3. Suppose that $f$ is not smooth, then $f$ has places of bad reduction, i.e., $C V(f)$ is non-empty. Then the result follows from Remark 2.4, Theorem 2.9, and Theorem 2.3.

Then Conjecture 2.1 follows from Theorem 2.12:

Theorem 2.13. The Bogomolov Conjecture holds if $\operatorname{char}(k)=0$. 


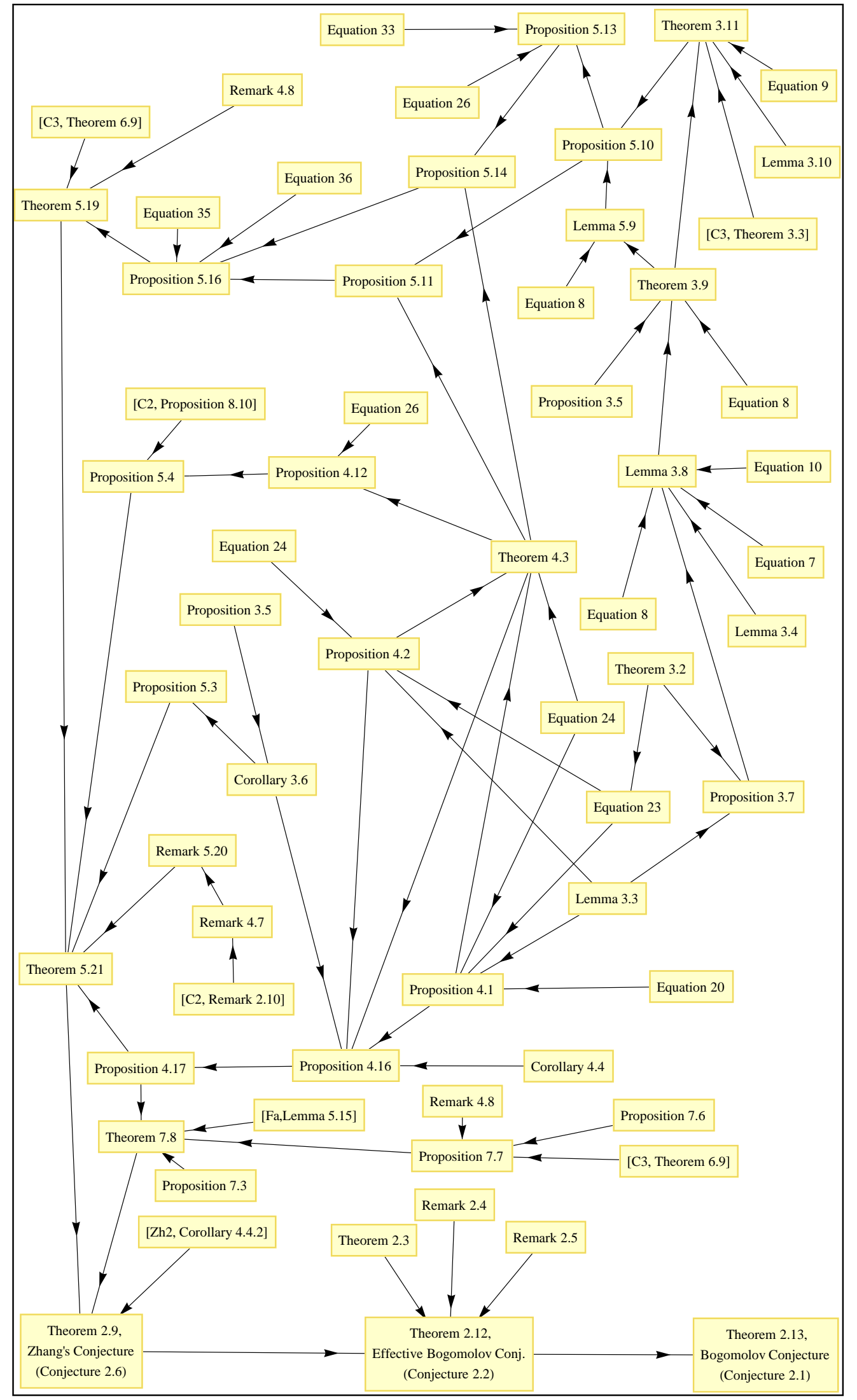

Figure 2. Major dependencies between the results that lead to the proofs of Conjectures (2.6), (2.2), and (2.1). 
The map of the results that lead to Theorem 2.13, Theorem 2.12 and Theorem 2.9 can be found in Figure 2.

If we consider the slightly different embedding $j_{D}: C(\bar{K}) \longrightarrow \operatorname{Jac}(C)(\bar{K})$ defined by $j_{D}(x)=x-D$ for a given $D \in \operatorname{Div}^{1}(C(\bar{K}))$, the Bogomolov Conjecture concerns the positivity of $\inf _{D \in D i v^{1}(C(\bar{K}))} a^{\prime}(D)$ when $f$ is non-isotrivial, where $a^{\prime}(D):=\lim _{x \in C(\bar{K})} \inf \hat{h}\left(j_{D}(x)\right)$ and $\hat{h}$ is the Néron-Tate height pairing on the Jacobian variety $\operatorname{Jac}(C)(\bar{K})=\operatorname{Pic}(C)(\bar{K})$ of the curve $C / K$. With this embedding, we can state Theorem 2.12 as follows:

Theorem 2.14. Let char $(k)=0$. If $f$ is non-isotrivial, then we have

$$
\inf _{D \in D i v^{1}(C(\bar{K}))} a^{\prime}(D) \geq \begin{cases}\frac{3}{\bar{g}-1}, & \text { if } f \text { is smooth } \\ \frac{1}{2(2 \bar{g}+1)}\left(t(\bar{g}) \delta_{0}(\Gamma)+\sum_{i \geq 1} \frac{2 i(\bar{g}-i)}{\bar{g}} \delta_{i}(\Gamma)\right), & \text { otherwise }\end{cases}
$$

with $t(\bar{g})$ as in Theorem [2.9. Therefore, the Effective Bogomolov Conjecture holds.

Theorem 2.14 shows that a stronger version of Faber's Conjecture [Fa, 1.3] holds.

Note that Theorem 2.12, Theorem 2.13 and Theorem 2.14 will also extend to the positive characteristic case if the following conjecture holds:

Conjecture 2.15. (Grothendieck, Gillet-Soulé) [GS] Let $k$ be a number field or a function field with positive characteristic, then the following height inequality holds:

$$
\left\langle\Delta_{\xi}, \Delta_{\xi}\right\rangle \geq 0
$$

Moreover, it becomes an equality precisely when $\Delta_{\xi}$ is rationally equivalent to 0.

\section{Metrized GRAPHS AND THEIR TAU CONSTANTS}

Rumely introduced metrized graphs to study arithmetic properties of curves and developed arithmetic capacity theory. T. Chinburg and Rumely [CR] used metrized graphs when they introduced their "capacity pairing". Another pairing satisfying "desirable" properties is the "admissible pairing on curves" introduced by Zhang [Zh1]. Metrized graphs were used as a non-archimedean analogue of a Riemann surface ([Ru], $\mathrm{CR}]$, and [Zh1]). Following Zhang's approach, A. Moriwaki used metrized graphs and Green's functions to prove specific cases of Bogomolov's conjecture over function fields in a series of papers, [AM1, AM2, and [AM3]. Extending Moriwaki's approach, Yamaki [KY1] proved very special cases of effective generalized Bogomolov's conjecture over function fields.

Metrized graphs arise as dual graphs of curves. Chinburg and Rumely [CR] worked with a canonical measure $\mu_{c a n}$ of total mass 1 on a metrized graph $\Gamma$. Similarly, Zhang worked with a measure $\mu_{a d}$ of total mass 1 on $\Gamma$. The measure $\mu_{a d}$ defined in $\$ 4$ is a generalization of $\mu_{\text {can }}$ defined in this section.

A metrized graph $\Gamma$ is a finite connected graph equipped with a distinguished parametrization of each of its edges. In particular, $\Gamma$ is a one-dimensional manifold except at finitely many "branch points". See also the articles [Ru] and [Zh1].

A metrized graph can have multiple edges and self-loops. For any given $p \in \Gamma$, the number of directions emanating from $p$ will be called the valence of $p$, and will be denoted by $v(p)$. By definition, there can be only finitely many $p \in \Gamma$ with $v(p) \neq 2$.

Given a metrized graph $\Gamma$, we will denote its set of vertices by $V(\Gamma)$. We require that $V(\Gamma)$ is non-empty and that $p \in V(\Gamma)$ for each $p \in \Gamma$ with $v(p) \neq 2$. For a given metrized 
graph $\Gamma$, it is possible to enlarge the vertex set $V(\Gamma)$ by considering arbitrarily many valence 2 points as vertices.

For a given metrized graph $\Gamma$ with vertex set $V(\Gamma)$, the set of edges of $\Gamma$ is the set of closed line segments with end points in $V(\Gamma)$. We will denote the set of edges of $\Gamma$ by $E(\Gamma)$. However, if $e_{i}$ is an edge, by $\Gamma-e_{i}$ we mean the graph obtained by deleting the interior of $e_{i}$.

Let $v:=\#(V(\Gamma))$ and $e:=\#(E(\Gamma))$. We define the genus of $\Gamma$ to be the first Betti number $g(\Gamma):=e-v+1$ of the graph $\Gamma$. We will simply use $g$ to show $g(\Gamma)$ when there is no danger of confusion. Note that the genus is a topological invariant of $\Gamma$. In particular, it is independent of the choice of the vertex set $V(\Gamma)$. Since $\Gamma$ is connected, $g(\Gamma)$ coincides with the cyclotomic number of $\Gamma$ in combinatorial graph theory.

We denote the length of an edge $e_{i} \in E(\Gamma)$ by $L_{i}$. Then total length of $\Gamma$, which will be denoted by $\ell(\Gamma)$, is given by $\ell(\Gamma)=\sum_{i=1}^{e} L_{i}$.

The minimum number of vertices whose deletion disconnects $\Gamma$ is called the "vertex connectivity" of $\Gamma$ and will be denoted by $\kappa(\Gamma)$. The minimum number of edges whose deletion disconnects $\Gamma$ is called the "edge connectivity" of $\Gamma$ and will be denoted by $\Lambda(\Gamma)$. Let $\bar{\delta}(\Gamma):=\min \{v(p) \mid p \in V(\Gamma)\}$ be the minimum of valences of the vertices. Then by basic graph theory [BB, pg. 3], $\kappa(\Gamma) \leq \Lambda(\Gamma) \leq \bar{\delta}(\Gamma)$. We call a metrized graph $\Gamma$ irreducible, as in the article [Fa], if it can not be disconnected by deleting any single point. That is, $\Gamma$ has vertex connectivity at least 2 for each possible choice of vertex set $V(\Gamma)$. Therefore, if $\Gamma$ is irreducible, it has edge connectivity at least 2 . If the edge connectivity of a metrized graph $\Gamma$ is at least two, we also say that $\Gamma$ is a bridgeless metrized graph. It is clear from the definitions that every irreducible graph is bridgeless, but there can be bridgeless graphs which have vertex connectivity 1 and so are not irreducible. For example, union of two copies of the circle graph along a vertex is a bridgeless metrized graph but not an irreducible metrized graph.

Baker and Rumely $[\mathrm{BR}]$ defined the following measure valued Laplacian on a given metrized graph:

$$
\Delta_{x}(f(x))=-f^{\prime \prime}(x) d x-\sum_{p \in V(\Gamma)}\left[\sum_{\vec{v} \text { at } p} d_{\vec{v}} f(p)\right] \delta_{p}(x)
$$

for a continuous function $f: \Gamma \rightarrow \mathbb{C}$ such that $f$ is $\mathcal{C}^{2}$ on $\Gamma \backslash V(\Gamma)$ and $f^{\prime \prime}(x) \in L^{1}(\Gamma)$. See the article [BR, Section 4], for the description of $\operatorname{BDV}(\Gamma)$, the largest set of continuous functions for which $\Delta_{x}$ is defined.

In the article $\mathrm{CR}$, a kernel $j_{z}(x, y)$ giving a fundamental solution of the Laplacian is defined and studied as a function of $x, y, z \in \Gamma$. For fixed $z$ and $y$ it has the following physical interpretation: when $\Gamma$ is viewed as a resistive electric circuit with terminals at $z$ and $y$, with the resistance in each edge given by its length, then $j_{z}(x, y)$ is the voltage difference between $x$ and $z$, when unit current enters at $y$ and exits at $z$ (with reference voltage 0 at $z)$.

Lemma 3.1. [CR, Lemma 2.10] The function $j_{\zeta}(x, y)$ is symmetric in $x$ and in $y$, is jointly continuous as a function of all three variables, and is nonnegative, with $j_{\zeta}(\zeta, y)=j_{\zeta}(x, \zeta)=0$ for all $x, y, \zeta \in \Gamma$.

A self-contained proof of this fact is given in the article [Zh1]. 


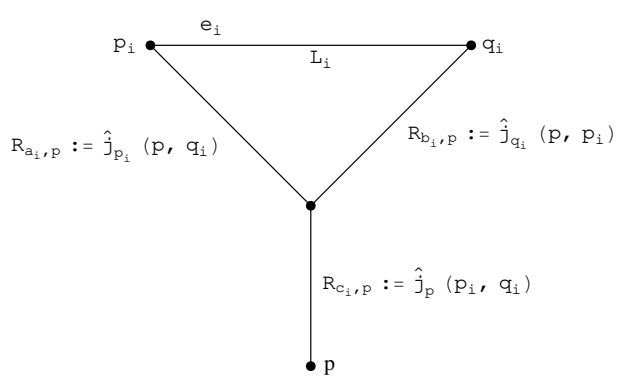

Figure 3. Circuit reduction with reference to an edge and a point.

The effective resistance between two points $x, y$ of a metrized graph $\Gamma$ is given by $r(x, y)=$ $j_{y}(x, x)$. We call $j_{z}(x, y)$ and $r(x, y)$ be the voltage function and the resistance function on $\Gamma$, respectively. The functions $j_{z}(x, y)$ and $r(x, y)$ are also studied in the articles $[\mathrm{BR}],[\mathrm{BF}]$, C1, [C2, C3, and C4.

We will denote by $R_{i}(\Gamma)$, or by $R_{i}$ if there is no danger of confusion, the resistance between the end points of an edge $e_{i}$ of a graph $\Gamma$ when the interior of the edge $e_{i}$ is deleted from $\Gamma$.

Let $\Gamma$ be a metrized graph with $p \in V(\Gamma)$, and let $e_{i} \in E(\Gamma)$ having end points $p_{i}$ and $q_{i}$. If $\Gamma-e_{i}$ is connected, then $\Gamma$ can be transformed to the graph in Figure 3 by circuit reductions. More details on this fact can be found in the articles [CR] and [C2, Section 2]. Note that in Figure 3, we have $R_{a_{i}, p}=\hat{j}_{p_{i}}\left(p, q_{i}\right), R_{b_{i}, p}=\hat{j}_{q_{i}}\left(p, p_{i}\right), R_{c_{i}, p}=\hat{j}_{p}\left(p_{i}, q_{i}\right)$, where $\hat{j}_{x}(y, z)$ is the voltage function in $\Gamma-e_{i}$. We have $R_{a_{i}, p}+R_{b_{i}, p}=R_{i}$ for each $p \in \Gamma$.

If $\Gamma-e_{i}$ is not connected, we set $R_{b_{i}, p}=R_{i}=\infty$ and $R_{a_{i}, p}=0$ if $p$ belongs to the component of $\Gamma-e_{i}$ containing $p_{i}$, and we set $R_{a_{i}, p}=R_{i}=\infty$ and $R_{b_{i}, p}=0$ if $p$ belongs to the component of $\Gamma-e_{i}$ containing $q_{i}$. We will use these notation in the rest of the paper.

For any real-valued, signed Borel measure $\mu$ on $\Gamma$ with $\mu(\Gamma)=1$ and $|\mu|(\Gamma)<\infty$, define the function $j_{\mu}(x, y)=\int_{\Gamma} j_{\zeta}(x, y) d \mu(\zeta)$. Clearly $j_{\mu}(x, y)$ is symmetric, and is jointly continuous in $x$ and $y$. Chinburg and Rumely [CR] discovered that there is a unique real-valued, signed Borel measure $\mu=\mu_{\text {can }}$ such that $j_{\mu}(x, x)$ is constant on $\Gamma$. The measure $\mu_{\text {can }}$ is called the canonical measure. See the articles [BR] and [C2 for several interpretations of $\mu_{\text {can }}$. Baker and Rumely $[\mathrm{BR}]$ called the constant $\frac{1}{2} j_{\mu}(x, x)$ the tau constant of $\Gamma$ and denoted it by $\tau(\Gamma)$.

Let $\mu$ be a real-valued signed Borel measure of total mass 1 on $\Gamma$. In the article [BR], the Arakelov-Green's function $g_{\mu}(x, y)$ associated to $\mu$ is defined to be $g_{\mu}(x, y)=\int_{\Gamma} j_{\zeta}(x, y) d \mu(\zeta)-$ $\int_{\Gamma} j_{\zeta}(x, y) d \mu(\zeta) d \mu(x) d \mu(y)$, where the latter integral is a constant that depends on $\Gamma$ and $\mu$.

As shown in the article [BR], $g_{\mu}(x, y)$ is continuous, symmetric (i.e., $g_{\mu}(x, y)=g_{\mu}(y, x)$, for each $x$ and $y$ ), and for each $y, \int_{\Gamma} g_{\mu}(x, y) d \mu(x)=0$. More precisely, as shown in the article [BR], one can characterize $g_{\mu}(x, y)$ as the unique function on $\Gamma \times \Gamma$ such that

(1) $g_{\mu}(x, y)$ is jointly continuous in $x, y$ and belongs to $\operatorname{BDV}_{\mu}(\Gamma)$ as a function of $x$, for each fixed $y$, where $\operatorname{BDV}_{\mu}(\Gamma):=\left\{f \in \operatorname{BDV}(\Gamma): \int_{\Gamma} f d \mu=0\right\}$.

(2) For fixed $y, g_{\mu}$ satisfies the identity $\Delta_{x} g_{\mu}(x, y)=\delta_{y}(x)-\mu(x)$.

(3) $\iint_{\Gamma \times \Gamma} g_{\mu}(x, y) d \mu(x) d \mu(y)=0$.

The diagonal values $g_{\mu_{c a n}}(x, x)$ are constant on $\Gamma$, and are equal to the tau constant $\tau(\Gamma)$. In terms of spectral theory, when $\Gamma$ has total length 1 , the tau constant is the trace of the inverse operator of $\Delta$. Note that the notation $\tau(\Gamma)$ is used in the article [Zh2, Equation 4.1.2] to denote another invariant of $\Gamma$. 
The following theorem gives an explicit description of $\mu_{\text {can }}$ :

Theorem 3.2. [CR, Theorem 2.11] For a given metrized graph $\Gamma$, let $L_{i}$ be the length of edge $e_{i} \in E(\Gamma)$, and let $R_{i}$ be the effective resistance between the endpoints of $e_{i}$ in the graph $\Gamma-e_{i}$. Then we have

$$
\mu_{\mathrm{can}}(x)=\sum_{p \in V(\Gamma)}\left(1-\frac{1}{2} v(p)\right) \delta_{p}(x)+\sum_{e_{i} \in E(\Gamma)} \frac{d x}{L_{i}+R_{i}} .
$$

The following two lemmas express $\tau(\Gamma)$ in terms of the resistance function and the canonical measure.

Lemma 3.3. REU For any metrized graph $\Gamma$ and its resistance function $r(x, y)$, and for each $x \in \Gamma, \tau(\Gamma)=\frac{1}{2} \int_{\Gamma} r(x, y) d \mu_{\text {can }}(y)$.

Lemma 3.4. [BR, Lemma 14.4] For any fixed $y \in \Gamma$, we have $\tau(\Gamma)=\frac{1}{4} \int_{\Gamma}\left(\frac{d}{d x} r(x, y)\right)^{2} d x$.

Lemma 3.4 implies that $\tau(\Gamma) \geq 0$ for any metrized graph $\Gamma$.

For the resistance function $r(x, y)$ in $\Gamma$, we use circuit reductions (parallel and series reductions, see the article [C2, Section 2] and the related references given therein) to obtain the following equalities:

$$
r\left(p_{i}, p\right)=\frac{\left(L_{i}+R_{b_{i}, p}\right) R_{a_{i}, p}}{L_{i}+R_{i}}+R_{c_{i}, p}, \quad \text { and } \quad r\left(q_{i}, p\right)=\frac{\left(L_{i}+R_{a_{i}, p}\right) R_{b_{i}, p}}{L_{i}+R_{i}}+R_{c_{i}, p} .
$$

Therefore,

$$
\begin{aligned}
& r\left(p_{i}, p\right)-r\left(q_{i}, p\right)=\frac{L_{i}\left(R_{a_{i}, p}-R_{b_{i}, p}\right)}{L_{i}+R_{i}}, \\
& r\left(p_{i}, p\right)+r\left(q_{i}, p\right)=\frac{L_{i} R_{i}}{L_{i}+R_{i}}+2 \frac{R_{a_{i}, p} R_{b_{i}, p}}{L_{i}+R_{i}}+2 R_{c_{i}, p} .
\end{aligned}
$$

The following proposition is obtained by evaluating the integral formula for the tau constant, given in Lemma 3.4, on each edges of $\Gamma$.

Proposition 3.5. [REU] Let $\Gamma$ be a metrized graph, and let $L_{i}$ be the length of the edge $e_{i}$, for $i \in\{1,2, \ldots, e\}$. Using the notation above, if we fix a vertex $p$ we have

$$
\tau(\Gamma)=\frac{1}{12} \sum_{e_{i} \in E(\Gamma)} \frac{L_{i}^{3}+3 L_{i}\left(R_{a_{i}, p}-R_{b_{i}, p}\right)^{2}}{\left(L_{i}+R_{i}\right)^{2}} .
$$

Here, if $\Gamma-e_{i}$ is not connected, i.e. $R_{i}$ is infinite, the summand corresponding to $e_{i}$ should be replaced by $3 L_{i}$, its limit as $R_{i} \longrightarrow \infty$.

The proof of Proposition 3.5 can be found in the article [C2, Proposition 2.9].

Corollary 3.6. Let $\Gamma$ be a circle graph. Then $\tau(\Gamma)=\frac{1}{12} \ell(\Gamma)$.

Proof. The result follows from Proposition 3.5 (equivalently, see the article [C2, Corollary 2.17]).

Chinburg and Rumely [CR, page 26] showed that

$$
\sum_{e_{i} \in E(\Gamma)} \frac{L_{i}}{L_{i}+R_{i}}=g, \quad \text { equivalently } \sum_{e_{i} \in E(\Gamma)} \frac{R_{i}}{L_{i}+R_{i}}=v-1 .
$$


The following proposition gives another formula for the tau constant which depends on the expression of $\mu_{\text {can }}(x)$ given in Theorem 3.2 .

Proposition 3.7. Let $\Gamma$ be a metrized graph and let $r(x, y)$ be the resistance function in $\Gamma$. Then for any $p \in V(\Gamma)$,

$$
\tau(\Gamma)=-\frac{1}{4} \sum_{q \in V(\Gamma)}(v(q)-2) r(p, q)+\frac{1}{2} \sum_{e_{i} \in E(\Gamma)} \frac{1}{L_{i}+R_{i}} \int_{0}^{L_{i}} r(p, x) d x .
$$

Proof. We have

$$
\begin{aligned}
& \tau(\Gamma)=\frac{1}{2} \int_{\Gamma} r(p, x) d \mu_{\mathrm{can}}(x), \quad \text { by Lemma 3.3, so by Theorem 3.2, } \\
& =\frac{1}{2} \sum_{q \in V(\Gamma)}\left(1-\frac{1}{2} v(p)\right) \int_{\Gamma} r(p, x) \delta_{q}(x)+\frac{1}{2} \sum_{e_{i} \in E(\Gamma)} \frac{1}{L_{i}+R_{i}} \int_{0}^{L_{i}} r(p, x) d x .
\end{aligned}
$$

Here, each edge $e_{i} \in E(\Gamma)$ is parametrized by a segment $\left[0, L_{i}\right]$, under its arclength parametrization. Thus the result follows.

The purpose of the following lemma is to clarify the relation between the formulas for $\tau(\Gamma)$ given in Proposition 3.5 and Proposition 3.7. This will help us to derive Theorem 3.9.

Lemma 3.8. Let $\Gamma$ be a bridgeless metrized graph, and let $p_{i}$ and $q_{i}$ be the end points of $e_{i} \in E(\Gamma)$. For any $p \in V(\Gamma)$, we have

$$
\begin{aligned}
\sum_{e_{i} \in E(\Gamma)} \frac{L_{i}\left(R_{a_{i}, p}-R_{b_{i}, p}\right)^{2}}{\left(L_{i}+R_{i}\right)^{2}} & =\sum_{e_{i} \in E(\Gamma)} \frac{L_{i}}{L_{i}+R_{i}}\left(r\left(p_{i}, p\right)+r\left(q_{i}, p\right)\right)-\sum_{q \in V(\Gamma)}(v(q)-2) r(p, q) \\
& =2 \sum_{q \in V(\Gamma)} r(p, q)-\sum_{e_{i} \in E(\Gamma)} \frac{R_{i}}{L_{i}+R_{i}}\left(r\left(p_{i}, p\right)+r\left(q_{i}, p\right)\right) .
\end{aligned}
$$

Proof. Let $p \in V(\Gamma)$. By Lemma 3.4, $4 \tau(\Gamma)=\int_{\Gamma}\left(\frac{d}{d x} r(p, x)\right)^{2} d x$. Thus, integration by parts gives

$$
4 \tau(\Gamma)=\left.\sum_{e_{i} \in E(\Gamma)}\left(r(p, x) \cdot \frac{d}{d x} r(p, x)\right)\right|_{0} ^{L_{i}}-\sum_{e_{i} \in E(\Gamma)} \int_{0}^{L_{i}} r(p, x) \frac{d^{2}}{d x^{2}} r(p, x) d x .
$$

If $x \in e_{i}$, then by parallel and series circuit reductions applied to the graph in Figure 3

$$
r(p, x)=\frac{\left(L_{i}-x+R_{b_{i}, p}\right)\left(x+R_{a_{i}, p}\right)}{L_{i}+R_{i}}+R_{c_{i}, p} \text {, so } \frac{d}{d x} r(p, x)=\frac{-2 x+L_{i}+R_{b_{i}, p}-R_{a_{i}, p}}{L_{i}+R_{i}} .
$$

Thus, $\frac{d^{2}}{d x^{2}} r(p, x)=\frac{-2}{L_{i}+R_{i}}$ if $x \in e_{i}$. This equality along with Proposition 3.7, Equations (10), (7), and (8) give the first equality in the theorem. Then the second equality holds by the following identity:

$$
\sum_{q \in V(\Gamma)} v(q) r(p, q)=\sum_{e_{i} \in E(\Gamma)}\left(r\left(p_{i}, p\right)+r\left(q_{i}, p\right)\right)
$$


Now we are ready to state a new formula for $\tau(\Gamma)$ which will play a crucial role in proving Conjecture 2.8 and so the related conjectures.

Theorem 3.9. Let $\Gamma$ be a bridgeless metrized graph, and let $r(x, y)$ be the resistance function on it. Then for any given $p \in V(\Gamma)$, we have

$$
\tau(\Gamma)=\frac{\ell(\Gamma)}{12}-\frac{1}{6} \sum_{q \in V(\Gamma)}(v(q)-2) r(p, q)+\frac{1}{3} \sum_{e_{i} \in E(\Gamma)} \frac{L_{i}}{L_{i}+R_{i}} R_{c_{i}, p} .
$$

Proof. By Equation (8) and the first equality in Lemma 3.8, we have

$$
\begin{aligned}
\sum_{e_{i} \in E(\Gamma)} \frac{L_{i}\left(R_{a_{i}, p}-R_{b_{i}, p}\right)^{2}}{\left(L_{i}+R_{i}\right)^{2}}= & \sum_{e_{i} \in E(\Gamma)} \frac{L_{i}^{2} R_{i}+2 L_{i} R_{a_{i}, p} R_{b_{i}, p}}{\left(L_{i}+R_{i}\right)^{2}}+2 \sum_{e_{i} \in E(\Gamma)} \frac{L_{i}}{L_{i}+R_{i}} R_{c_{i}, p} \\
& -\sum_{q \in V(\Gamma)}(v(q)-2) r(p, q) .
\end{aligned}
$$

By using $R_{i}=R_{a_{i}, p}+R_{b_{i}, p}$, we obtain $2 R_{a_{i}, p} R_{b_{i}, p}=\frac{1}{2} R_{i}^{2}-\frac{1}{2}\left(R_{a_{i}, p}-R_{b_{i}, p}\right)^{2} ;$ then substituting this in Equation (13) we obtain the following equality

$$
\begin{aligned}
3 \sum_{e_{i} \in E(\Gamma)} \frac{L_{i}\left(R_{a_{i}, p}-R_{b_{i}, p}\right)^{2}}{\left(L_{i}+R_{i}\right)^{2}}= & \sum_{e_{i} \in E(\Gamma)} \frac{2 L_{i}^{2} R_{i}+L_{i} R_{i}^{2}}{\left(L_{i}+R_{i}\right)^{2}}+4 \sum_{e_{i} \in E(\Gamma)} \frac{L_{i}}{L_{i}+R_{i}} R_{c_{i}, p} \\
& -2 \sum_{q \in V(\Gamma)}(v(q)-2) r(p, q) .
\end{aligned}
$$

Adding $\sum_{e_{i} \in E(\Gamma)} \frac{L_{i}^{3}}{\left(L_{i}+R_{i}\right)^{2}}$ to both sides of Equation (14) and using the fact that $\ell(\Gamma)=$ $\sum_{e_{i} \in E(\Gamma)} L_{i}$ gives

$$
\sum_{e_{i} \in E(\Gamma)} \frac{L_{i}^{3}+3 L_{i}\left(R_{a_{i}, p}-R_{b_{i}, p}\right)^{2}}{\left(L_{i}+R_{i}\right)^{2}}=\ell(\Gamma)+4 \sum_{e_{i} \in E(\Gamma)} \frac{L_{i}}{L_{i}+R_{i}} R_{c_{i}, p}-2 \sum_{q \in V(\Gamma)}(v(q)-2) r(p, q) .
$$

On the other hand the left hand side is equal to $12 \tau(\Gamma)$ by Proposition 3.5. Then the result follows.

For each $i \in\{1,2, \ldots e\}$, let $\bar{\Gamma}_{i}$ be the metrized graph obtained from a metrized graph $\Gamma$ by contracting the i-th edge $e_{i} \in E(\Gamma)$ to its end points. If $e_{i} \in \Gamma$ has end points $p_{i}$ and $q_{i}$, then in $\bar{\Gamma}_{i}$, these points become an identical vertex which we will denote as $\bar{p}_{i}$. Note that $\ell(\Gamma)=\ell\left(\bar{\Gamma}_{i}\right)+L_{i}$. We will denote the valence of $p \in \bar{\Gamma}_{i}$ by $\bar{v}_{i}(p)$. If $q \in V(\Gamma)-\left\{p_{i}, q_{i}\right\}$, then $q \in V\left(\bar{\Gamma}_{i}\right)$ and $\bar{v}_{i}(q)=v(q)$. If $e_{i}$ is not a self loop, then $\bar{v}_{i}\left(\bar{p}_{i}\right)=v\left(p_{i}\right)+v\left(q_{i}\right)-2$.

Lemma 3.10. Let $\Gamma$ be a metrized graph with an edge $e_{i} \in E(\Gamma)$ such that $\Gamma-e_{i}$ is connected, and let $\bar{\Gamma}_{i}$ be defined as before. Let $r(x, y)$ and $r_{i}(x, y)$ be the resistance functions in $\Gamma$ and $\bar{\Gamma}_{i}$, respectively. Then we have

$$
\sum_{q \in V\left(\bar{\Gamma}_{i}\right)}\left(\bar{v}_{i}(q)-2\right) r_{i}\left(\bar{p}_{i}, q\right)=\sum_{q \in V(\Gamma)}(v(q)-2)\left(\frac{R_{a_{i}, q} R_{b_{i}, q}}{R_{i}}+R_{c_{i}, q}\right) .
$$




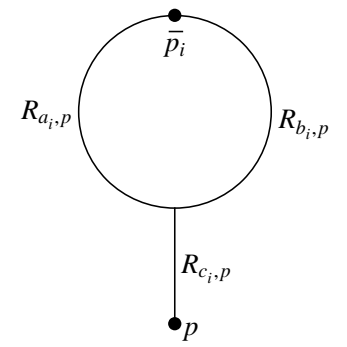

FIGURE 4. $\bar{\Gamma}_{i}$ after circuit reductions.

Proof. When $e_{i}$ is contracted, $\Gamma$ shown in Figure 3 becomes $\bar{\Gamma}_{i}$ shown in Figure 4 , Therefore, by applying parallel circuit reduction and using the fact that $R_{a_{i}, q}+R_{b_{i}, q}=R_{i}$ for each $q \in V(\Gamma)$ we obtain the following equality:

$$
r_{i}\left(\bar{p}_{i}, q\right)=\frac{R_{a_{i}, q} R_{b_{i}, q}}{R_{i}}+R_{c_{i}, q}
$$

Equation (15) and the fact that $\bar{v}_{i}(q)=v(q)$ for each $q \in V(\Gamma)-\left\{p_{i}, q_{i}\right\}$ yields the following equality for each $q \in V(\Gamma)-\left\{p_{i}, q_{i}\right\}$ :

$$
\left(\bar{v}_{i}(q)-2\right) r_{i}\left(\bar{p}_{i}, q\right)=(v(q)-2)\left(\frac{R_{a_{i}, q} R_{b_{i}, q}}{R_{i}}+R_{c_{i}, q}\right) .
$$

On the other hand, $R_{a_{i}, q} \cdot R_{b_{i}, q}=0=R_{c_{i}, q}$ for $q \in\left\{p_{i}, q_{i}\right\}$, and $r_{i}\left(\bar{p}_{i}, \bar{p}_{i}\right)=0$. Thus the result follows from Equation (16).

Next, by using Lemma 3.10 and several other results about $\tau(\Gamma)$ we will prove the following theorem which will be useful in proving the second inequality in Conjecture 2.8 .

Theorem 3.11. Let $\Gamma$ be a bridgeless metrized graph with $\#(V(\Gamma))=v \geq 3$. Let $R_{a_{i}, q}$, $R_{b_{i}, q}, R_{i}$ and $R_{c_{i}, q}$ be as defined before for each $e_{i} \in E(\Gamma)$, and let $\bar{R}_{j}$ and $\bar{R}_{c_{j}, \bar{p}_{i}}$ be defined similarly for each $e_{j} \in E\left(\bar{\Gamma}_{i}\right)$. Then we have

$$
\begin{gathered}
\tau(\Gamma)=\frac{\ell(\Gamma)}{12}-\frac{1}{6(v-2)} \sum_{q \in V(\Gamma)}(v(q)-2) \sum_{e_{i} \in E(\Gamma)} \frac{R_{a_{i}, q} R_{b_{i}, q}+R_{i} R_{c_{i}, q}}{L_{i}+R_{i}} \\
+\frac{1}{3(v-2)} \sum_{e_{i} \in E(\Gamma)} \frac{R_{i}}{L_{i}+R_{i}} \sum_{e_{j} \in E\left(\bar{\Gamma}_{i}\right)} \frac{L_{j} \bar{R}_{c_{j}, \bar{p}_{i}}}{L_{j}+\bar{R}_{j}} .
\end{gathered}
$$

Proof. Since $\Gamma$ is bridgeless, $\bar{\Gamma}_{i}$ is also bridgeless for each $e_{i} \in E(\Gamma)$. Thus, we can apply Theorem 3.9 for each $\bar{\Gamma}_{i}$ with vertex $\bar{p}_{i}$ to obtain,

$$
\tau\left(\bar{\Gamma}_{i}\right)=\frac{\ell\left(\bar{\Gamma}_{i}\right)}{12}-\frac{1}{6} \sum_{q \in V\left(\bar{\Gamma}_{i}\right)}\left(\bar{v}_{i}(q)-2\right) r_{i}\left(\bar{p}_{i}, q\right)+\frac{1}{3} \sum_{e_{j} \in E\left(\bar{\Gamma}_{i}\right)} \frac{L_{j} \bar{R}_{c_{j}, \bar{p}_{i}}}{L_{j}+\bar{R}_{j}}
$$



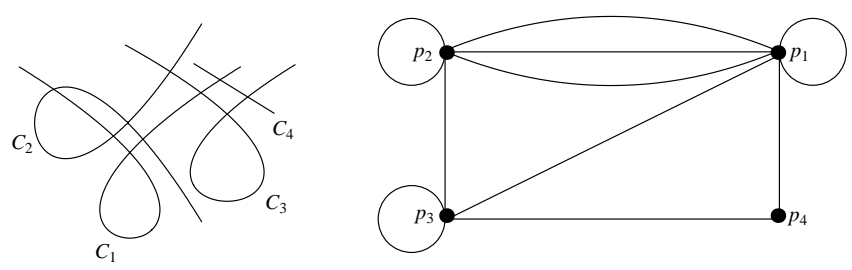

FiguRE 5. A graph and its dual graph

where $r_{i}\left(\bar{p}_{i}, q\right)$ is the resistance, in $\bar{\Gamma}_{i}$, between the vertices $\bar{p}_{i}$ and $q$. Then we multiply both sides of Equation (17) by $\frac{R_{i}}{L_{i}+R_{i}}$ and sum over all edges $e_{i} \in E(\Gamma)$ to obtain

$$
\begin{aligned}
\sum_{e_{i} \in E(\Gamma)} \frac{R_{i} \tau\left(\bar{\Gamma}_{i}\right)}{L_{i}+R_{i}}=\frac{1}{12} & \sum_{e_{i} \in E(\Gamma)} \frac{R_{i} \ell\left(\bar{\Gamma}_{i}\right)}{L_{i}+R_{i}}-\frac{1}{6} \sum_{e_{i} \in E(\Gamma)} \frac{R_{i}}{L_{i}+R_{i}} \sum_{q \in V\left(\bar{\Gamma}_{i}\right)}\left(\bar{v}_{i}(q)-2\right) r_{i}\left(\bar{p}_{i}, q\right) \\
& +\frac{1}{3} \sum_{e_{i} \in E(\Gamma)} \frac{R_{i}}{L_{i}+R_{i}} \sum_{e_{j} \in E\left(\bar{\Gamma}_{i}\right)} \frac{L_{j} \bar{R}_{c_{j}, \bar{p}_{i}}}{L_{j}+\bar{R}_{j}}
\end{aligned}
$$

On the other hand, it follows from the article [C3, Theorem 3.3] that

$$
\sum_{e_{i} \in E(\Gamma)} \frac{R_{i} \tau\left(\bar{\Gamma}_{i}\right)}{L_{i}+R_{i}}=(v-2) \tau(\Gamma)+\frac{1}{12} \sum_{e_{i} \in E(\Gamma)} \frac{L_{i}^{2}}{L_{i}+R_{i}}
$$

Multiply both sides of the equation given in Lemma 3.10 by $\frac{R_{i}}{L_{i}+R_{i}}$ and sum over all edges $e_{i} \in E(\Gamma)$ to obtain

$$
\sum_{e_{i} \in E(\Gamma)} \frac{R_{i}}{L_{i}+R_{i}} \sum_{q \in V\left(\bar{\Gamma}_{i}\right)}\left(\bar{v}_{i}(q)-2\right) r_{i}\left(\bar{p}_{i}, q\right)=\sum_{q \in V(\Gamma)}(v(q)-2) \sum_{e_{i} \in E(\Gamma)} \frac{R_{a_{i}, q} R_{b_{i}, q}+R_{i} R_{c_{i}, q}}{L_{i}+R_{i}}
$$

The result follows by substituting Equation (19) and Equation (20) into Equation (18), using the fact that $\ell\left(\bar{\Gamma}_{i}\right)=\ell(\Gamma)-L_{i}$, and using Equation (9).

\section{Polarized METRIZED GRAPHS}

In this section, following the articles [Zh2] and [Fa], we first introduce the notion of a polarized metrized graph and related concepts. Then we give formulas for several invariants $\epsilon(\Gamma), \varphi(\Gamma), a(\Gamma)$, and $\lambda(\Gamma)$. These invariants will be important in proving Conjecture 2.8 . Finally, we prove the first inequality in Conjecture 2.8 and derive a formula for $\varphi(\Gamma)$ which will be used in $\$ 5$ to prove the second inequality in Conjecture 2.8 .

Let $\Gamma$ be a metrized graph and let $\mathbf{q}: \Gamma \rightarrow \mathbb{N}$ be a function on the set of vertices of $\Gamma$. The canonical divisor $K$ of $(\Gamma, \mathbf{q})$ is defined to be the following divisor on $\Gamma$ :

$$
K=\sum_{p \in V(\Gamma)}(v(p)-2+2 \mathbf{q}(p)) p, \quad \text { and } \quad \delta_{K}(x)=\sum_{p \in V(\Gamma)}(v(p)-2+\mathbf{q}(p)) \delta_{p}(x) .
$$

The pair $(\Gamma, \mathbf{q})$ will be called a polarized metrized graph (pm-graph in short) if $\mathbf{q}$ is nonnegative and $K$ is an effective divisor. The genus $\bar{g}(\Gamma)$ of a pm-graph $(\Gamma, \mathbf{q})$ is defined to 
be

$$
\bar{g}(\Gamma)=1+\frac{1}{2} \operatorname{deg} K=g(\Gamma)+\sum_{p \in V(\Gamma)} \mathbf{q}(p) .
$$

We will simply use $\bar{g}$ to show $\bar{g}(\Gamma)$ when there is no danger of confusion. Note that $\bar{g} \geq 1$ for a pm-graph. We call a pm-graph $(\Gamma, \mathbf{q})$ irreducible if the underlying metrized graph $\Gamma$ is irreducible.

Note that the reduction graph $R(X)$ of any semistable curve $X$ of genus $\bar{g}$ over a discrete valuation ring is a pm-graph of genus $\bar{g}$.

Recall that how pm-graphs are obtained from fibres of a semistable fibration $f: X \longrightarrow Y$ is explained at page 2 .

Let $\mu_{a d}(x)$ be the admissible metric associated to $K$ (as defined in the article [Zh1, Lemma 3.7]). Then

$$
\mu_{a d}(x)=\frac{1}{\bar{g}}\left(\sum_{p \in V(\Gamma)} \mathbf{q}(p) \delta_{p}(x)+\sum_{i \in E(\Gamma)} \frac{d x}{L_{i}+R_{i}}\right) .
$$

Then by Theorem $\underline{3.2}$, we have

$$
\mu_{a d}(x)=\frac{1}{2 \bar{g}}\left(2 \mu_{c a n}(x)+\delta_{K}(x)\right) .
$$

Moreover, $\delta_{K}(\Gamma)=\operatorname{deg}(K)=2 \bar{g}-2$, and by Equation (9) $\mu_{c a n}(\Gamma)=1=\mu_{a d}(\Gamma)$.

Set $\theta(\Gamma):=\sum_{p, q \in V(\Gamma)}(v(p)-2+2 \mathbf{q}(p))(v(q)-2+2 \mathbf{q}(q)) r(p, q)$, and define

$$
\begin{aligned}
& \epsilon(\Gamma)=\iint_{\Gamma \times \Gamma} r(x, y) \delta_{K}(x) \mu_{a d}(x), \\
& a(\Gamma)=\frac{1}{2} \iint_{\Gamma \times \Gamma} r(x, y) \mu_{a d}(x) \mu_{a d}(y), \\
& \varphi(\Gamma)=3 \bar{g} \cdot a(\Gamma)-\frac{1}{4}(\epsilon(\Gamma)+\ell(\Gamma)), \\
& \lambda(\Gamma)=\frac{\bar{g}-1}{6(2 \bar{g}+1)} \varphi(\Gamma)+\frac{1}{12}(\epsilon(\Gamma)+\ell(\Gamma)) .
\end{aligned}
$$

We have $\theta(\Gamma) \geq 0$ for any pm-graph $\Gamma$, since the corresponding canonical divisor $K$ is effective.

Note that the second invariant in Equation (25) was denoted as $\tau(\Gamma)$ in the article [Zh2]. In order not to have notational conflict with the articles [BR], [C1], [C2], [C3], and [C4], we denote it by $a(\Gamma)$. For a pm-graph $(\Gamma, \mathbf{q})$ by $\tau(\Gamma)$ we mean the tau constant of the underlying metrized graph $\Gamma$.

Proposition 4.1. Let $\Gamma$ be a pm-graph. Then we have

$$
\epsilon(\Gamma)=\frac{(4 \bar{g}-4) \tau(\Gamma)}{\bar{g}}+\frac{\theta(\Gamma)}{2 \bar{g}} .
$$


Proof. By Equation (25), we have

$$
\begin{aligned}
\epsilon(\Gamma) & =\iint_{\Gamma \times \Gamma} r(x, y) \delta_{K}(x) \mu_{a d}(x) . \text { Then by Equation (21) } \\
& =\sum_{p \in V(\Gamma)}(v(p)-2+2 \mathbf{q}(p)) \int_{\Gamma} r(p, y) \mu_{a d}(y), \quad \text { and by Equation (24) } \\
& =\sum_{p \in V(\Gamma)}(v(p)-2+2 \mathbf{q}(p)) \int_{\Gamma} r(p, y)\left(\frac{1}{2 \bar{g}}\left(2 \mu_{c a n}(y)+\delta_{K}(y)\right)\right.
\end{aligned}
$$

Then the result follows by Lemma 3.3 and the fact that $\operatorname{deg}(K)=2 \bar{g}-2$.

Recall that both $\tau(\Gamma)$ and $\theta(\Gamma)$ are nonnegative for any pm-graph $\Gamma$. Therefore, Proposition 4.1 implies that $\epsilon(\Gamma) \geq 0$. Similarly, $a(\Gamma) \geq 0$ for any pm-graph $\Gamma$ by the following proposition.

Proposition 4.2. Let $\Gamma$ be a pm-graph. Then we have

$$
a(\Gamma)=\frac{(2 \bar{g}-1) \tau(\Gamma)}{\bar{g}^{2}}+\frac{\theta(\Gamma)}{8 \bar{g}^{2}} .
$$

Proof. By Equation (25), we have

$$
\begin{aligned}
2 a(\Gamma) & =\iint_{\Gamma \times \Gamma} r(x, y) \mu_{a d}(x) \mu_{a d}(y) \text {. Then by Equation (24), } \\
& =\int_{\Gamma}\left(\int_{\Gamma} r(x, y) \frac{1}{2 \bar{g}}\left(2 \mu_{c a n}(x)+\delta_{K}(x)\right)\right) \mu_{a d}(y) \\
& =\int_{\Gamma}\left(\frac{2 \tau(\Gamma)}{\bar{g}}+\frac{1}{2 \bar{g}} \sum_{p \in V(\Gamma)}(v(p)-2+2 \mathbf{q}(p)) r(p, y)\right) \mu_{a d}(y), \quad \text { by Lemma 3.3. } \\
& =\frac{2 \tau(\Gamma)}{\bar{g}}+\frac{1}{2 \bar{g}} \sum_{p \in V(\Gamma)}(v(p)-2+2 \mathbf{q}(p)) \int_{\Gamma} r(p, y) \mu_{a d}(y), \quad \text { since } \mu_{a d}(\Gamma)=1 .
\end{aligned}
$$

Since $\operatorname{deg}(K)=2 \bar{g}-2$, the result follows by a similar calculation done to obtain the third equality from the first equality.

Theorem 4.3. Let $\Gamma$ be a pm-graph. Then we have

$$
\varphi(\Gamma)=\frac{(5 \bar{g}-2) \tau(\Gamma)}{\bar{g}}+\frac{\theta(\Gamma)}{4 \bar{g}}-\frac{\ell(\Gamma)}{4} .
$$

Proof. By Equation (25), we have

$$
\begin{aligned}
\varphi(\Gamma) & =3 \bar{g}(\Gamma) \cdot a(\Gamma)-\frac{1}{4}(\epsilon(\Gamma)+\ell(\Gamma)) \text {. Then by Proposition } 4.2 \text { and Proposition } 4.1 \\
& =3 \bar{g}\left(\frac{(2 \bar{g}-1) \tau(\Gamma)}{\bar{g}^{2}}+\frac{\theta(\Gamma)}{8 \bar{g}^{2}}\right)-\frac{1}{4}\left(\frac{(4 \bar{g}-4) \tau(\Gamma)}{\bar{g}}+\frac{\theta(\Gamma)}{2 \bar{g}}+\ell(\Gamma)\right) .
\end{aligned}
$$

This is equivalent to what we wanted.

Corollary 4.4. Let $\Gamma$ be a pm-graph. Then we have

$$
\lambda(\Gamma)=\frac{(3 \bar{g}-3) \tau(\Gamma)}{4 \bar{g}+2}+\frac{\theta(\Gamma)}{16 \bar{g}+8}+\frac{(\bar{g}+1) \ell(\Gamma)}{16 \bar{g}+8} .
$$


Proof. By substituting the formula for $\varphi(\Gamma)$ given in Theorem 4.3 and the formula for $\epsilon(\Gamma)$ given in Proposition 4.1 into formula for $\lambda(\Gamma)$ given in Equation (25), we obtain the result.

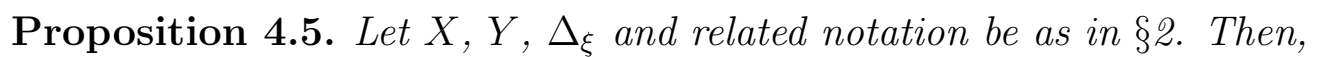

$$
\omega_{X / Y}^{2}=\frac{2 \bar{g}-2}{2 \bar{g}+1}\left\langle\Delta_{\xi}, \Delta_{\xi}\right\rangle+\frac{18(\bar{g}-1)}{2 \bar{g}+1} \sum_{i=1}^{s} \tau\left(\Gamma_{y_{i}}\right)+\frac{3}{4 \bar{g}+2} \sum_{i=1}^{s} \theta\left(\Gamma_{y_{i}}\right)-\frac{\bar{g}-1}{4 \bar{g}+2} \delta .
$$

Proof. By Equation (2) and Equation (3),

$$
\omega_{X / Y}^{2}=\frac{2 \bar{g}-2}{2 \bar{g}+1}\left\langle\Delta_{\xi}, \Delta_{\xi}\right\rangle+\frac{2 \bar{g}-2}{2 \bar{g}+1} \sum_{i=1}^{s} \varphi\left(\Gamma_{y_{i}}\right)+\sum_{i=1}^{s} \epsilon\left(\Gamma_{y_{i}}\right) .
$$

Then the result follows from Theorem 4.3, Proposition 4.1 and the fact that $\delta=\sum_{i=1}^{s} \ell\left(\Gamma_{y_{i}}\right)$ (see page 2).

Remark 4.6. A proper upper bound for $\omega_{X / Y}^{2}$ implies the Effective Mordell conjecture. Note that $\tau(\Gamma) \leq \frac{1}{4} \ell(\Gamma)$ for a pm-graph $\Gamma$ containing bridges. Rumely showed that this inequality is sharp for a pm-graph $\Gamma$ with no cycles (i.e., the corresponding metrized graph has genus $0)$. If $\Gamma$ is a bridgeless pm-graph, then $\tau(\Gamma) \leq \frac{1}{12} \ell(\Gamma)$ by [C2, Corollary 5.8], which is sharp for a circle graph as shown in Corollary [3.6. Moreover, $\theta(\Gamma) \leq 8(\bar{g}-1)^{2} \tau(\Gamma)$ for a pm-graph $\Gamma$ with genus $\bar{g} \geq 2$ by [C1, Theorem 4.18].

Remark 4.7. Let $\Gamma$ be a graph with a vertex set $\# V(\Gamma)$. If we enlarge $\# V(\Gamma)$ by considering more valence 2 points $p \in \Gamma$ assigned with $\boldsymbol{q}(p)=0$ as vertices, then we have the following observations:

The tau constant $\tau(\Gamma)$ does not change, by its valence property [C2, Remark 2.10]. The resistance function on $\Gamma$ does not change. The genus $\bar{g}$ remains the same. Moreover, $v(p)-$ $2+2 \boldsymbol{q}(p)=0$ for each new vertex $p$. Therefore, $\varphi(\Gamma)$ does not change. We call this property the valence property of $\varphi(\Gamma)$.

Remark 4.8. For any given pm-graph $\Gamma$, if we multiply each edge length by a constant $t$, then the tau constant $\tau(\Gamma)$ and $r(x, y)$ for each $x$ and $y$ in $\Gamma$ change by a factor of $t$ [C2, Remark 2.15]. This implies that $\varphi(\Gamma)=\varphi\left(\Gamma^{N}\right) \ell(\Gamma)$ for any pm-graph $\Gamma$, where $\Gamma^{N}$ is the pm-graph obtained from $\Gamma$ by dividing each edge length in $E(\Gamma)$ by $\ell(\Gamma)$. We call this property the scale-independence of $\varphi(\Gamma)$.

Lemma 4.9 shows how the first inequality in Conjecture 2.8 can be expressed in terms of invariants of bridgeless pm-graphs.

Lemma 4.9. The first inequality in Conjecture 2.8 is equivalent to the following inequality:

$$
\ell(\Gamma) \leq 12 \tau(\Gamma)+\frac{\theta(\Gamma)}{\bar{g}-1}
$$

Proof. Both $a(\Gamma)$ and $\epsilon(\Gamma)$ are expressed in terms of $\tau(\Gamma)$ and $\theta(\Gamma)$ in Proposition 4.2 and Proposition 4.1, respectively. After substituting these values into the first inequality in Conjecture 2.8 and doing some algebra, we obtain the result in the Lemma.

Lemma 4.10 shows that proving Conjecture 2.6 is enough to show that the second equality in Conjecture 2.8 holds. 
Lemma 4.10. The second inequality in Conjecture 2.8 is equivalent to the following inequality:

$$
\varphi(\Gamma) \geq \frac{c(\bar{g}) \ell(\Gamma)}{4}
$$

Proof. The result follows by arguments similar to those in the proof of Lemma 4.9, using Theorem 4.3,

Theorem 4.11. Let $\Gamma$ be a bridgeless pm-graph. Then we have

$$
\frac{\bar{g}-1}{\bar{g}+1}(\ell(\Gamma)-4 \bar{g} \cdot a(\Gamma)) \leq \epsilon(\Gamma) .
$$

In particular, the first inequality in Conjecture 2.8 holds.

Proof. Recall that $\epsilon(\Gamma) \geq 0$ for any pm-graph $\Gamma$. Thus the inequality clearly holds when $\bar{g}=1$.

Suppose $\bar{g} \geq 2$. We first multiply both sides of the equality in Theorem 3.9 by $(v(p)-$ $2+2 \mathbf{q}(p))$. Then we sum the resulting equality over all $p \in V(\Gamma)$, and use the fact that $\operatorname{deg}(K)=2 \bar{g}-2$. In this way, we obtain

$$
\begin{gathered}
(2 \bar{g}-2) \tau(\Gamma)=\frac{(2 \bar{g}-2) \ell(\Gamma)}{12}-\frac{1}{6} \sum_{p, q \in V(\Gamma)}(v(q)-2)(v(p)-2+2 \mathbf{q}(p)) r(p, q) \\
+\frac{1}{3} \sum_{p \in V(\Gamma)}(v(p)-2+2 \mathbf{q}(p)) \sum_{e_{i} \in E(\Gamma)} \frac{L_{i}}{L_{i}+R_{i}} R_{c_{i}, p} .
\end{gathered}
$$

Since $(v(q)-2)=(v(q)-2+2 \mathbf{q}(q)-2 \mathbf{q}(q))$,

$$
\begin{gathered}
(2 \bar{g}-2) \tau(\Gamma)=\frac{(2 \bar{g}-2) \ell(\Gamma)}{12}-\frac{\theta(\Gamma)}{6}+\frac{1}{3} \sum_{p, q \in V(\Gamma)}(v(p)-2+2 \mathbf{q}(p)) \mathbf{q}(q) r(p, q) \\
+\frac{1}{3} \sum_{p \in V(\Gamma)}(v(p)-2+2 \mathbf{q}(p)) \sum_{e_{i} \in E(\Gamma)} \frac{L_{i}}{L_{i}+R_{i}} R_{c_{i}, p}
\end{gathered}
$$

Equivalently,

$$
\begin{aligned}
12 \tau(\Gamma)+\frac{\theta(\Gamma)}{\bar{g}-1} & =\ell(\Gamma)+\frac{2}{\bar{g}-1} \sum_{p, q \in V(\Gamma)}(v(p)-2+2 \mathbf{q}(p)) \mathbf{q}(q) r(p, q) \\
& +\frac{2}{\bar{g}-1} \sum_{p \in V(\Gamma)}(v(p)-2+2 \mathbf{q}(p)) \sum_{e_{i} \in E(\Gamma)} \frac{L_{i}}{L_{i}+R_{i}} R_{c_{i}, p} .
\end{aligned}
$$

Since $K$ is effective and $\mathbf{q}(p) \geq 0$ for each $p \in V(\Gamma)$, Equation (27) implies that

$$
12 \tau(\Gamma)+\frac{\theta(\Gamma)}{\bar{g}-1} \geq \ell(\Gamma)
$$

Therefore, the inequality we wanted to show follows from Equation (28) and Lemma 4.9.

Recall that an irreducible metrized graph is bridgeless. Hence, the first inequality in Conjecture 2.8 holds. 
Proposition 4.12. Let $\Gamma$ be a bridgeless pm-graph. Then we have

$$
\begin{gathered}
\varphi(\Gamma)=\frac{(2 \bar{g}+1) \tau(\Gamma)}{\bar{g}}-\frac{\ell(\Gamma)}{4 \bar{g}}+\frac{1}{2 \bar{g}} \sum_{p, q \in V(\Gamma)}(v(p)-2+2 \boldsymbol{q}(p)) \boldsymbol{q}(q) r(p, q) \\
+\frac{1}{2 \bar{g}} \sum_{p \in V(\Gamma)}(v(p)-2+2 \boldsymbol{q}(p)) \sum_{e_{i} \in E(\Gamma)} \frac{L_{i}}{L_{i}+R_{i}} R_{c_{i}, p} .
\end{gathered}
$$

In particular,

$$
\varphi(\Gamma) \geq \frac{(2 \bar{g}+1) \tau(\Gamma)}{\bar{g}}-\frac{\ell(\Gamma)}{4 \bar{g}} .
$$

Proof. By multiplying both sides of Equation (27) by $\frac{\bar{g}-1}{4 \bar{g}}$ and using Theorem 4.3, we obtain the equality. Since $K$ is effective and $\mathbf{q}$ is non-negative, the inequality follows.

Lemma 4.9, the proof of Theorem 4.11 and Proposition 4.13 below clarify the relation between Conjecture 2.7 and the first inequality in Conjecture 2.8.

Proposition 4.13. Let $\Gamma$ be a bridgeless pm-graph. Then we have

$$
\begin{array}{r}
\lambda(\Gamma)=\frac{\bar{g}}{8 \bar{g}+4} \ell(\Gamma)+\frac{1}{8 \bar{g}+4} \sum_{p, q \in V(\Gamma)}(v(p)-2+2 \boldsymbol{q}(p)) \boldsymbol{q}(q) r(p, q) \\
+\frac{1}{8 \bar{g}+4} \sum_{p \in V(\Gamma)}(v(p)-2+2 \boldsymbol{q}(p)) \sum_{e_{i} \in E(\Gamma)} \frac{L_{i}}{L_{i}+R_{i}} R_{c_{i}, p} .
\end{array}
$$

In particular,

$$
\lambda(\Gamma) \geq \frac{\bar{g}}{8 \bar{g}+4} \ell(\Gamma)
$$

Proof. If we substitute the value of $\varphi(\Gamma)$ given in Proposition 4.12 and the value of $\epsilon(\Gamma)$ given in Proposition 4.1 into the formula for $\lambda(\Gamma)$ given in Equation (25), we obtain the following equality:

$$
\begin{aligned}
\lambda(\Gamma)=\frac{\bar{g}-1}{2 \bar{g}} \tau(\Gamma)+\frac{\theta(\Gamma)}{24 \bar{g}}+\frac{4 \bar{g}^{2}+\bar{g}+1}{24 \bar{g}(2 \bar{g}+1)} \ell(\Gamma) \\
\quad+\frac{\bar{g}-1}{12 \bar{g}(2 \bar{g}+1)} \sum_{p, q \in V(\Gamma)}(v(p)-2+2 \mathbf{q}(p)) \mathbf{q}(q) r(p, q) \\
\quad+\frac{\bar{g}-1}{12 \bar{g}(2 \bar{g}+1)} \sum_{p \in V(\Gamma)}(v(p)-2+2 \mathbf{q}(p)) \sum_{e_{i} \in E(\Gamma)} \frac{L_{i}}{L_{i}+R_{i}} R_{c_{i}, p} .
\end{aligned}
$$

The equality in the proposition follows by multiplying Equation (27) by $\frac{\bar{g}-1}{24 \bar{g}}$ and using Equation (29). Since $\Gamma$ is a pm-graph, the associated canonical divisor $K$ is effective. That is, $v(p)-2+2 \mathbf{q}(p) \geq 0$ and $\mathbf{q}(p) \geq 0$ for each $p \in V(\Gamma)$. Hence, the inequality in the proposition follows.

Corollary 4.14. Let $(\Gamma, \boldsymbol{q})$ be a bridgeless pm-graph with $\boldsymbol{q} \equiv 0$. If $\Gamma$ has one or two vertices, then we have

$$
\lambda(\Gamma)=\frac{\bar{g}}{8 \bar{g}+4} \ell(\Gamma)
$$



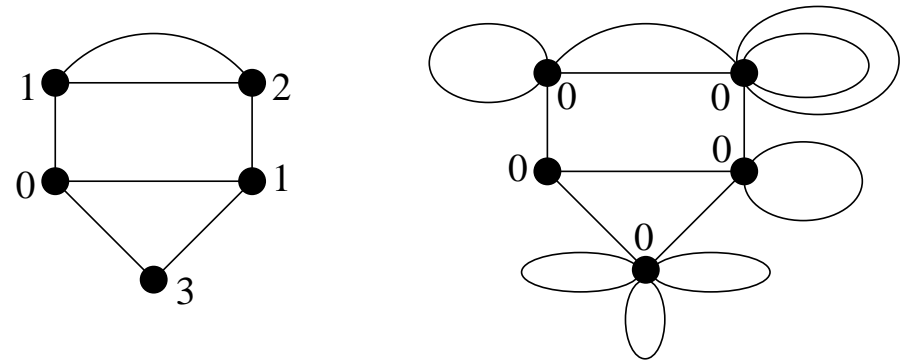

Figure $6 . \Gamma$ and $\Gamma_{0}$. Values of $\mathbf{q}$ and $\mathbf{q}_{0}$ are shown in each graph.

Proof. Let $V(\Gamma)=\{p, q\}$. We have $R_{c_{i}, p}=0=R_{c_{i}, q}$, so the result follows by Proposition 4.13 .

Remark 4.15. Whenever a pm-graph $(\Gamma, 0)$ has two vertices say $p$ and $q$, we have $R_{c_{i}, p}=$ $R_{c_{i}, q}=0$. Therefore, the lower bound for $\lambda(\Gamma)$ in Proposition 4.13 is sharp. However, if $\Gamma$ has more than 3 vertices, the bound can be far from sharp, as shown in Proposition 5.23; and $\lambda(\Gamma)$ can be expressed as in Proposition 5.15.

In order to investigate the role of the case $\mathbf{q} \equiv 0$ in finding lower bounds for $\varphi(\Gamma), \lambda(\Gamma)$, $\epsilon(\Gamma)$, and $a(\Gamma)$, we make the following construction:

Let $(\Gamma, \mathbf{q})$ be a pm-graph of genus $\bar{g} \geq 2$. If there is a vertex $p \in V(\Gamma)$ with $\mathbf{q}(p)>0$, we attach $\mathbf{q}(p)$ circles of length $\varepsilon>0$ to $\Gamma$ at the vertex $p$. By repeating this process for each such vertex, we obtain a new metrized graph, which we denote by $\Gamma_{0}$. By choosing $\mathbf{q}_{0}=0$ as the polarization on $\Gamma_{0}$, we have a pm-graph $\left(\Gamma_{0}, 0\right)$. Figure 6 shows an example. Note that $V\left(\Gamma_{0}\right)=V(\Gamma), \ell\left(\Gamma_{0}\right)=\ell(\Gamma)+\varepsilon \sum_{p \in V(\Gamma)} \mathbf{q}(p)$. Since $g\left(\Gamma_{0}\right)=g(\Gamma)+\sum_{p \in V(\Gamma)} \mathbf{q}(p)$, $\bar{g}\left(\Gamma_{0}\right)=\bar{g}(\Gamma)$ by Equation (22) . Moreover,

$$
\begin{aligned}
v_{\Gamma_{0}}(p) & =v_{\Gamma}(p)+2 \mathbf{q}(p), \quad \text { for each } p \in V(\Gamma), \text { and } \\
r_{\Gamma_{0}}(p, q) & =r_{\Gamma}(p, q), \quad \text { for each } p \text { and } q \text { in } V(\Gamma),
\end{aligned}
$$

where $v_{\Gamma}(p)$ is valence of $p$ in $\Gamma$, and $r_{\Gamma}(x, y)$ is the resistance function on $\Gamma$. Equation (30) implies $\theta\left(\Gamma_{0}\right)=\theta(\Gamma)$. Moreover, $\tau\left(\Gamma_{0}\right)=\tau(\Gamma)+\frac{\varepsilon}{12} \sum_{p \in V(\Gamma)} \mathbf{q}(p)$ which can be seen by applying the additive property of the tau constant [C2, page 11] and using the fact that $\tau(\beta)=\frac{\ell(\beta)}{12}$ for a circle graph $\beta$ (see Corollary 3.6). Thus, the following proposition follows from Theorem 4.3, Proposition 4.1, Proposition 4.2, and Corollary 4.4.

Proposition 4.16. Let $\Gamma$ be an arbitrary pm-graph with genus $\bar{g}$, and let $\Gamma_{0}$ be as defined above. If $Q=\sum_{p \in V(\Gamma)} \boldsymbol{q}(p)$, we have

$$
\begin{array}{ll}
\varphi\left(\Gamma_{0}\right)=\varphi(\Gamma)+\varepsilon Q \frac{\bar{g}-1}{6 \bar{g}}, & \epsilon\left(\Gamma_{0}\right)=\epsilon(\Gamma)+\varepsilon Q \frac{\bar{g}-1}{3 \bar{g}} \\
a\left(\Gamma_{0}\right)=a(\Gamma)+\varepsilon Q \frac{2 \bar{g}-1}{12 \bar{g}^{2}}, & \lambda\left(\Gamma_{0}\right)=\lambda(\Gamma)+\varepsilon Q \frac{\bar{g}}{8 \bar{g}+4} .
\end{array}
$$

We obtain the following proposition by choosing an appropriate $\varepsilon$ as we construct $\Gamma_{0}$.

Proposition 4.17. Let $(\Gamma, \boldsymbol{q})$ be a given pm-graph with genus $\bar{g}$. For any given $\varepsilon>0$, there exist a pm-graph $\left(\Gamma_{0}, 0\right)$ of genus $\bar{g}$ such that

$$
\varphi\left(\Gamma_{0}\right) \leq \varphi(\Gamma)+\varepsilon, \quad \epsilon\left(\Gamma_{0}\right) \leq \epsilon(\Gamma)+\varepsilon, \quad a\left(\Gamma_{0}\right) \leq a(\Gamma)+\varepsilon, \quad \lambda\left(\Gamma_{0}\right) \leq \lambda(\Gamma)+\varepsilon .
$$


Remark 4.18. Since $\varepsilon$ in Proposition 4.17 can be taken arbitrarily small for a given pmgraph $\Gamma$, it will be enough to consider pm-graphs with polarization $\boldsymbol{q} \equiv 0$ in order to give lower bounds for $\varphi(\Gamma), \epsilon(\Gamma)$, a $(\Gamma)$, and $\lambda(\Gamma)$.

Note that Proposition 4.17 should be compared with the article [Fa, Lemma 5.14] which gives a similar result for $\varphi(\Gamma)$.

\section{Simple POlARIZED METRIZED GRAPHS}

In this section, we will prove the second inequality in Conjecture 2.8, which is equivalent to a lower bound for $\varphi(\Gamma)$ by Lemma 4.10. We will call a pm-graph $(\Gamma, \mathbf{q})$ a simple pm-graph if $\mathbf{q} \equiv 0$. We will denote a simple pm-graph $(\Gamma, 0)$ simply by $\Gamma$ when there is no danger of confusion. Note that $\bar{g}=g$ (i.e., $\bar{g}(\Gamma)=g(\Gamma)$ ) when $\mathbf{q}(p)=0$ for each $p \in V(\Gamma$ ) (see Equation (22) ). To show that $\varphi(\Gamma)$ and $\lambda(\Gamma)$ are bounded by positive constants depending only on the genus and the length of the metrized graph, it will be enough to consider irreducible simple pm-graphs by [Zh2, 4.4.2], by our discussion about irreducible graphs in $\$ 3$ and by Proposition 4.17. Related results can be found in the article [Fa, Lemmas 5.12, 5.14 and 5.15].

Theorem 5.1. Let $\Gamma$ be a bridgeless simple pm-graph with $\#(V(\Gamma))=v$ and $\#(E(\Gamma))=e$.

Then we have

$$
\varphi(\Gamma)=\frac{(2 g+1) \tau(\Gamma)}{g}-\frac{\ell(\Gamma)}{4 g}+\frac{1}{2 g} \sum_{p \in V(\Gamma)}(v(p)-2) \sum_{e_{i} \in E(\Gamma)} \frac{L_{i}}{L_{i}+R_{i}} R_{c_{i}, p} .
$$

Moreover,
(i) $\varphi(\Gamma) \geq \frac{(2 g+1) \tau(\Gamma)}{g}-\frac{\ell(\Gamma)}{4 g}$,
(ii) $\varphi(\Gamma) \geq \frac{(g-1) \ell(\Gamma)}{12 g(g+1)}$
(iii) $\varphi(\Gamma) \geq \frac{(4 e-5 v) \ell(\Gamma)}{4 g(v+6)}$,
(iv) $\varphi(\Gamma) \geq \frac{(g-1) \ell(\Gamma)}{4 g(g+2)}$
(v) $\varphi(\Gamma) \geq \frac{(2 g+1) g^{2} v+6(2 g+1)(v-1)^{2}-3 e^{2} v}{12 g \cdot v \cdot e^{2}} \ell(\Gamma)$, if all edge lengths are equal

to each other and $v(p) \geq 3$ for each $p \in V(\Gamma)$.

$$
\text { (vi) } \varphi(\Gamma) \geq \frac{2 g+1}{12 g}\left(1-\frac{4}{\Lambda(\Gamma)}\right)^{2} \ell(\Gamma)+\frac{4(2 g+1)(\Lambda(\Gamma)-2) \ell(\Gamma)}{g(v+6) \Lambda(\Gamma)^{2}}-\frac{\ell(\Gamma)}{4 g} \text {, if } \Lambda(\Gamma) \geq 4 \text {. }
$$

Therefore, the second inequality in Conjecture 2.8 holds.

Proof. Since $\mathbf{q}(p)=0$ for each $p \in V(\Gamma)$, the formula for $\varphi(\Gamma)$ in Proposition 4.12 reduces to the formula given in the theorem.

Proof of $(i)$ :

It is given that $\Gamma$ is a pm-graph, so the associated canonical divisor $K$ is effective. That is, in this case we have $v(p)-2 \geq 0$ for each $p \in V(\Gamma)$. This gives $(i)$.

Proof of $(i i)$ :

We have $\tau(\Gamma) \geq \frac{\ell(\Gamma)}{6(g+1)}$ by [C3, Corollary 3.7]. Then the result follows from part $(i)$.

Proof of $($ iii):

We have $\tau(\Gamma) \geq \frac{\ell(\Gamma)}{2(v+6)}$ by $[$ 33, Theorem 6.10 part $(2)]$. Then we have $\varphi(\Gamma) \geq \frac{(2 g+1) \ell(\Gamma)}{2 g(v+6)}-\frac{\ell(\Gamma)}{4 g}$ by $(i)$ and we finish by using the fact that $g=e-v+1$. 
Proof of $(i v)$ : Let $V(\Gamma)$ be a vertex set such that $v(p) \geq 3$ if $p \in \Gamma$. Note that such a vertex set can be found by Remark 4.7 for any bridgeless simple graph with genus at least two. Then by basic graph theory, $e \geq \frac{3 v}{2}$, where $e$ and $v$ are the number of edges and the number of vertices, respectively. Thus, $2(g-1) \geq v$. Then the result follows from $(i i i)$.

Proof of $(v)$ :

When the edges have equal lengths and $v(p) \geq 3$ for each $p \in V(\Gamma)$, we have $\tau(\Gamma) \geq$ $\left(\frac{1}{12}\left(\frac{g}{e}\right)^{2}+\frac{1}{2 v}\left(\frac{v-1}{e}\right)^{2}\right) \ell(\Gamma)$ by [C3, Theorem 6.11]. Then the result follows from part $(i)$.

Proof of $(v i)$ :

When $\Lambda(\Gamma) \geq 4, \tau(\Gamma) \geq \frac{1}{12}\left(1-\frac{4}{\Lambda(\Gamma)}\right)^{2} \ell(\Gamma)+\frac{4 \ell(\Gamma)(\Lambda(\Gamma)-2)}{(v+6) \Lambda(\Gamma)^{2}}$ by [C3, Theorem 6.10 part $\left.(1)\right]$. Then the result follows from part $(i)$.

Proof of the last part:

Using Lemma 4.10, proof of the second inequality in Conjecture 2.8 follows from any of parts (ii) and $(i v)$.

Note that when $g \geq 4$, Theorem 5.19 gives bounds to $\varphi(\Gamma)$ that are much stronger than the bounds given in Theorem 5.1 ,

Remark 5.2. The proof of Conjecture 2.8 follows from Theorem 4.11 and Theorem 5.1.

Suppose $\Gamma$ be a simple pm-graph with $V(\Gamma)=\{p\}$ and $\#(E(\Gamma))=e \geq 2$. We call such a graph a bouquet graph. When $e=2, \Gamma$ is just a union of two circles along $p$.

Proposition 5.3. Let $\Gamma$ be a simple bouquet graph. Then we have

$$
\varphi(\Gamma)=\frac{g-1}{6 g} \ell(\Gamma)
$$

Proof. The tau constant for a circle graph $\beta$ is $\frac{\ell(\beta)}{12}$ by Corollary 3.6 . Then by the additivity of the tau constant [C2, page 11] $\tau(\Gamma)=\frac{\ell(\Gamma)}{12}$. In this case, $R_{c_{i}, p}=0$ for any edge $e_{i} \in E(\Gamma)$. Since $\Gamma$ is a simple pm-graph, which means $\mathbf{q}(p)=0$ for each $p \in V(\Gamma)$, the result follows from the formula of $\varphi(\Gamma)$ in Proposition 4.12 .

Suppose $\Gamma$ be a simple pm-graph with $V(\Gamma)=\{p, q\}$ and $\#(E(\Gamma))=e \geq 2$. We call such a graph a banana graph. When $e=2, \Gamma$ is just a circle graph with two vertices. Note that $g(\Gamma)=e-1$ for a banana graph with $e$ edges.

Proposition 5.4. Let $\Gamma$ be a simple banana graph with e edges, and let the set of edge lengths be indexed by $\left\{L_{1}, L_{2}, \ldots, L_{e}\right\}$. Then we have

$$
\varphi(\Gamma)=\frac{g-1}{6 g} \ell(\Gamma)-\frac{(g-1)(2 g+1)}{6 g \sum_{i=1}^{e} \frac{1}{L_{i}}} .
$$

In particular,

$$
\varphi(\Gamma) \geq \frac{g(g-1)}{6(g+1)^{2}} \ell(\Gamma) .
$$

Proof. The tau constant for banana graphs are calculated explicitly in the article [C2, Proposition 8.10]. Namely, for a banana graph $\Gamma, \tau(\Gamma)=\frac{\ell(\Gamma)}{12}-\frac{e-2}{6 \sum_{i=1}^{e} \frac{1}{L_{i}}}$. Since $\frac{\sum_{i=1}^{e} L_{i}}{e} \geq \frac{e}{\sum_{i=1}^{e} \frac{1}{L_{i}}}$ by Arithmetic-Harmonic Mean inequality, we have $\tau(\Gamma) \geq\left(\frac{1}{12}-\frac{e-2}{6 e^{2}}\right) \ell(\Gamma)$. In this case, $R_{c_{i}, q}=R_{c_{i}, p}=0$ for any edge $e_{i} \in E(\Gamma)$. Since $\Gamma$ is a simple pm-graph, the result follows from the formula of $\varphi(\Gamma)$ in Proposition 4.12 . 
When $\Gamma$ be a simple graph with two vertices, a formula for $\varphi(\Gamma)$ can be given explicitly by using Proposition 5.4 and the additivity of $\varphi(\Gamma)$.

The following corollary shows that Theorem 5.1 verifies or improves the previously known lower bounds to $\varphi(\Gamma)$ when $g \leq 4$.

Corollary 5.5. Let $\Gamma$ be a bridgeless simple pm-graph. Then $\varphi(\Gamma) \geq c(g) \cdot \ell(\Gamma)$, where $c(g)$ can be taken as $\frac{1}{27}$ if $g=2, \frac{1}{30}$ if $g=3, \frac{1}{32}$ if $g=4$, and $\frac{1}{35}$ if $g=5$.

Proof. We have $\varphi(\Gamma) \geq \frac{(g-1) \ell(\Gamma)}{4 g(g+2)}$ by Theorem 5.1 part $(i v)$. Evaluation of this lower bound for $g=3,4,5$ gives the desired lower bounds.

To obtain the given lower bound when $g=2$, we work with a vertex set such that $v(p) \geq 3$ if $p \in V(\Gamma)$. Then, as discussed in the proof of part $(i v)$ in Theorem 5.1, we have $2(g-1) \geq v$. Therefore, $v \leq 2$ if $g=2$. Then either $\Gamma$ is the union of two circles along a point or a banana graph. Then the proof follows from Proposition 5.3 and Proposition 5.4.

Remark 5.6. We recovered the known bound for $\varphi(\Gamma)$ when $g=2$; and improved the known bounds when $g=3,4$. Note that the given lower bounds are sharp only for $g=2$.

If any two distinct vertices of a graph $\Gamma$ are connected by one and only one edge and if there are no self loops, we call $\Gamma$ be a complete graph. For a complete graph on $v$ vertices, the valence of any vertex is $v-1$, and so by basic graph theory $e=\frac{v(v-1)}{2}$, and $g=\frac{(v-1)(v-2)}{2}$.

Proposition 5.7. Let $\Gamma$ be a simple complete pm-graph on v vertices, where $v>3$. If all the edge lengths are equal, then we have

$$
\varphi(\Gamma)=\frac{(v-2)(v-3)\left(v^{2}+6 v-6\right)}{6 v^{3}(v-1)} \ell(\Gamma) .
$$

In particular, $\frac{17}{288} \ell(\Gamma) \leq \varphi(\Gamma) \leq \frac{9499}{54925} \ell(\Gamma)$.

Proof. By [C2, Proposition 2.16], $\tau(\Gamma)=\left(\frac{1}{12}\left(1-\frac{2}{v}\right)^{2}+\frac{2}{v^{3}}\right) \ell(\Gamma)$. By arguments given in the proof of [C2, Proposition 2.16], $r(p, q)=\frac{v-1}{e^{2}}$ for each pair of distinct vertices $p, q \in$ $V(\Gamma)$. Therefore, $\theta(\Gamma)=(v-3)^{2} \sum_{p, q \in V(\Gamma)} r(p, q)=\frac{4(v-3)^{2}}{v}$. In addition, $e=\frac{v(v-1)}{2}$ and $g=\frac{(v-1)(v-2)}{2}$. Substituting these values into the formula for $\varphi(\Gamma)$ in Theorem 4.3 gives the result. Then by elementary calculus we find that the minimum of $\varphi(\Gamma)$ is attained at $v=4$ and that its maximum is attained at $v=26$.

By using the formula for $\lambda(\Gamma)$ given in Corollary 4.4 and following the arguments as in the proof of Proposition 5.7, we can calculate $\lambda(\Gamma)$ for a simple complete pm-graph $\Gamma$.

Proposition 5.8. Let $\Gamma$ be a simple complete pm-graph on $v$ vertices, where $v>3$. If all the edge lengths are equal, then we have

$$
\lambda(\Gamma)=\frac{\left(v^{3}+v^{2}-12 v+18\right)(v-2)}{8 v^{2}\left(v^{2}-3 v+3\right)} \ell(\Gamma)=\frac{g}{8 g+4}+\frac{(g+2) \sqrt{8 g+1}-7 g-2}{(2 g+1)(3+\sqrt{8 g+1})^{2}} \ell(\Gamma) .
$$

In particular, $\frac{25}{224} \ell(\Gamma) \leq \lambda(\Gamma) \leq \frac{499}{3650} \ell(\Gamma)$, where the minimum is attained at $v=4$ and the maximum is attained at $v=10$.

In view of Proposition 5.8, there is not much room in improving the slope inequality given in (4). 
We obtained a formula for $\theta(\Gamma)$ in Equation (27). The following Lemma gives another formula for $\theta(\Gamma)$. These two formulas will play important roles in giving an improved lower bound for $\varphi(\Gamma)$, which will give another proof of Conjecture 2.6 and the second inequality in Conjecture 2.8.

Lemma 5.9. Let $\Gamma$ be a bridgeless simple pm-graph with genus $g$ and $\#(V(\Gamma))=v$. Then we have

$$
\begin{gathered}
\theta(\Gamma)=(2 g-2) \sum_{e_{i} \in E(\Gamma)} \frac{L_{i} R_{i}}{L_{i}+R_{i}}+2 \sum_{q \in V(\Gamma)}(v(q)-2) \sum_{e_{i} \in E(\Gamma)} \frac{R_{a_{i}, q} R_{b_{i}, q}+R_{i} R_{c_{i}, q}}{L_{i}+R_{i}} \\
+2 \sum_{q \in V(\Gamma)}(v(q)-4) \sum_{e_{i} \in E(\Gamma)} \frac{L_{i} R_{c_{i}, q}}{L_{i}+R_{i}}+12 v \cdot \tau(\Gamma)-v \cdot \ell(\Gamma) .
\end{gathered}
$$

Proof. First, we note the following equality for any given $q \in V(\Gamma)$.

$$
\begin{aligned}
\sum_{p \in V(\Gamma)} v(p) r(p, q) & =\sum_{e_{i} \in E(\Gamma)}\left(r\left(p_{i}, q\right)+r\left(q_{i}, q\right)\right), \quad p_{i}, q_{i} \text { are end points of } e_{i} . \\
& \left.=\sum_{e_{i} \in E(\Gamma)} \frac{L_{i} R_{i}}{L_{i}+R_{i}}+2 \sum_{e_{i} \in E(\Gamma)}\left(\frac{R_{a_{i}, q} R_{b_{i}, q}}{L_{i}+R_{i}}+R_{c_{i}, q}\right), \quad \text { by Equation (8) }\right) .
\end{aligned}
$$

Summing the equality in Theorem 3.9 over all vertices gives

$$
2 \sum_{p, q \in V(\Gamma)}(v(q)-2) r(p, q)=v \cdot \ell(\Gamma)-12 v \cdot \tau(\Gamma)+4 \sum_{p \in V(\Gamma)} \sum_{e_{i} \in E(\Gamma)} \frac{L_{i} R_{c_{i}, p}}{L_{i}+R_{i}} .
$$

On the other hand,

$$
\begin{aligned}
\theta(\Gamma) & =\sum_{p, q \in V(\Gamma)}(v(q)-2)(v(p)-2) r(p, q), \quad \text { since } \Gamma \text { is simple. } \\
& =\sum_{q \in V(\Gamma)}(v(q)-2) \sum_{p \in V(\Gamma)} v(p) r(p, q)-2 \sum_{p, q \in V(\Gamma)}(v(q)-2) r(p, q) .
\end{aligned}
$$

Then, the result follows by substituting Equations (31) and (32) into Equation (33) and using the fact that $\sum_{p \in V(\Gamma)}(v(p)-2)=2 g-2$.

Next, we will combine Theorem 3.11 and Lemma $\$ 5.9$ to obtain a new formula for $\theta(\Gamma)$.

Proposition 5.10. Let $\Gamma$ be a bridgeless simple pm-graph with genus $g$ and with at least three vertices. Then we have

$$
\begin{aligned}
\theta(\Gamma)=- & 2 \ell(\Gamma)+24 \tau(\Gamma)+(2 g-2) \sum_{e_{i} \in E(\Gamma)} \frac{L_{i} R_{i}}{L_{i}+R_{i}}+4 \sum_{e_{i} \in E(\Gamma)} \frac{R_{i}}{L_{i}+R_{i}} \sum_{e_{j} \in E\left(\bar{\Gamma}_{i}\right)} \frac{L_{j} \bar{R}_{c_{j}, \bar{p}_{i}}}{L_{j}+\bar{R}_{j}} \\
& +2 \sum_{q \in V(\Gamma)}(v(q)-4) \sum_{e_{i} \in E(\Gamma)} \frac{L_{i} R_{c_{i}, q}}{L_{i}+R_{i}} .
\end{aligned}
$$


Proof. Let $\#(V(\Gamma))=v$. We multiply both sides of the equality given in Theorem 3.11 by $12(v-2)$. This will give us

$$
\begin{array}{r}
2 \sum_{q \in V(\Gamma)}(v(q)-2) \sum_{e_{i} \in E(\Gamma)} \frac{R_{a_{i}, q} R_{b_{i}, q}+R_{i} R_{c_{i}, q}}{L_{i}+R_{i}}=4 \sum_{e_{i} \in E(\Gamma)} \frac{R_{i}}{L_{i}+R_{i}} \sum_{e_{j} \in E\left(\bar{\Gamma}_{i}\right)} \frac{L_{j} \bar{R}_{c_{j}, \bar{p}_{i}}}{L_{j}+\bar{R}_{j}} \\
+(v-2) \ell(\Gamma)-12(v-2) \tau(\Gamma) .
\end{array}
$$

Then we substitute this into Lemma 5.9 to obtain the result.

Proposition 5.11. Let $\Gamma$ be a bridgeless simple pm-graph with genus $g$ and with at least three vertices. Then we have

$$
\begin{gathered}
\varphi(\Gamma)=\frac{5 g+4}{g} \tau(\Gamma)-\frac{g+2}{4 g} \ell(\Gamma)+\frac{g-1}{2 g} \sum_{e_{i} \in E(\Gamma)} \frac{L_{i} R_{i}}{L_{i}+R_{i}}+\frac{1}{g} \sum_{e_{i} \in E(\Gamma)} \frac{R_{i}}{L_{i}+R_{i}} \sum_{e_{j} \in E\left(\bar{\Gamma}_{i}\right)} \frac{L_{j} \bar{R}_{c_{j}, \bar{p}_{i}}}{L_{j}+\bar{R}_{j}} \\
+\frac{1}{2 g} \sum_{q \in V(\Gamma)}(v(q)-4) \sum_{e_{i} \in E(\Gamma)} \frac{L_{i} R_{c_{i}, q}}{L_{i}+R_{i}} .
\end{gathered}
$$

In particular, if $v(q) \geq 4$ for each $q \in V(\Gamma)$, then we have

$$
\varphi(\Gamma) \geq \frac{5 g+4}{g} \tau(\Gamma)-\frac{g+2}{4 g} \ell(\Gamma)+\frac{g-1}{2 g} \sum_{e_{i} \in E(\Gamma)} \frac{L_{i} R_{i}}{L_{i}+R_{i}} .
$$

Proof. We obtain the equality by substituting the formula for $\theta(\Gamma)$ given in Proposition 5.10 into the formula for $\varphi(\Gamma)$ given in Theorem 4.3. Since all of $R_{c_{i}, q}, L_{i}, R_{i}, \bar{R}_{c_{j}, \bar{p}_{i}}$, and $\bar{R}_{j}$ are positive, the inequality follows from the equality if $v(q) \geq 4$ for each $q \in V(\Gamma)$.

Similarly, by using Proposition 5.10 and Corollary 4.4, we obtain the following expression for $\lambda(\Gamma)$.

Proposition 5.12. Let $\Gamma$ be a bridgeless simple pm-graph with genus $g$ and with at least three vertices. Then we have

$$
\begin{aligned}
\lambda(\Gamma)= & \frac{3 g+3}{4 g+2} \tau(\Gamma)+\frac{g-1}{16 g+8} \ell(\Gamma)+\frac{g-1}{8 g+4} \sum_{e_{i} \in E(\Gamma)} \frac{L_{i} R_{i}}{L_{i}+R_{i}} \\
& +\frac{1}{4 g+2} \sum_{e_{i} \in E(\Gamma)} \frac{R_{i}}{L_{i}+R_{i}} \sum_{e_{j} \in E\left(\bar{\Gamma}_{i}\right)} \frac{L_{j} \bar{R}_{c_{j}, \bar{p}_{i}}}{L_{j}+\bar{R}_{j}}+\frac{1}{8 g+4} \sum_{q \in V(\Gamma)}(v(q)-4) \sum_{e_{i} \in E(\Gamma)} \frac{L_{i} R_{c_{i}, q}}{L_{i}+R_{i}} .
\end{aligned}
$$

In particular, if $v(q) \geq 4$ for each $q \in V(\Gamma)$, then we have

$$
\lambda(\Gamma) \geq \frac{3 g+3}{4 g+2} \tau(\Gamma)+\frac{g-1}{16 g+8} \ell(\Gamma)+\frac{g-1}{8 g+4} \sum_{e_{i} \in E(\Gamma)} \frac{L_{i} R_{i}}{L_{i}+R_{i}} .
$$

The following is another formula for $\theta(\Gamma)$. 
Proposition 5.13. Let $\Gamma$ be a bridgeless simple pm-graph with genus $g$ and with at least three vertices. Then we have

$$
\begin{aligned}
\theta(\Gamma)= & \frac{g-3}{2} \ell(\Gamma)-6(g-3) \tau(\Gamma)+(g-1) \sum_{e_{i} \in E(\Gamma)} \frac{L_{i} R_{i}}{L_{i}+R_{i}}+2 \sum_{e_{i} \in E(\Gamma)} \frac{R_{i}}{L_{i}+R_{i}} \sum_{e_{j} \in E\left(\bar{\Gamma}_{i}\right)} \frac{L_{j} \bar{R}_{c_{j}, \bar{p}_{i}}}{L_{j}+\bar{R}_{j}} \\
& +2 \sum_{q \in V(\Gamma)}(v(q)-3) \sum_{e_{i} \in E(\Gamma)} \frac{L_{i} R_{c_{i}, q}}{L_{i}+R_{i}} .
\end{aligned}
$$

Proof. Since $\Gamma$ is simple, Equation (27) can be expressed as follows:

$$
\theta(\Gamma)=(g-1) \ell(\Gamma)-12(g-1) \tau(\Gamma)+2 \sum_{p \in V(\Gamma)}(v(p)-2) \sum_{e_{i} \in E(\Gamma)} \frac{L_{i} R_{c_{i}, p}}{L_{i}+R_{i}} .
$$

Then the result is obtained by adding the formulas for $\theta(\Gamma)$ given in Proposition 5.10 and Equation (34).

Proposition 5.14. Let $\Gamma$ be a bridgeless simple pm-graph with genus $g$ and at least three vertices. Then we have

$$
\begin{aligned}
\varphi(\Gamma)= & \frac{7 g+5}{2 g} \tau(\Gamma)-\frac{g+3}{8 g} \ell(\Gamma)+\frac{g-1}{4 g} \sum_{e_{i} \in E(\Gamma)} \frac{L_{i} R_{i}}{L_{i}+R_{i}}+\frac{1}{2 g} \sum_{e_{i} \in E(\Gamma)} \frac{R_{i}}{L_{i}+R_{i}} \sum_{e_{j} \in E\left(\bar{\Gamma}_{i}\right)} \frac{L_{j} \bar{R}_{c_{j}, \bar{p}_{i}}}{L_{j}+\bar{R}_{j}} \\
& +\frac{1}{2 g} \sum_{q \in V(\Gamma)}(v(q)-3) \sum_{e_{i} \in E(\Gamma)} \frac{L_{i} R_{c_{i}, q}}{L_{i}+R_{i}} .
\end{aligned}
$$

In particular, if $v(q) \geq 3$ for each $q \in V(\Gamma)$, then we have

$$
\varphi(\Gamma) \geq \frac{7 g+5}{2 g} \tau(\Gamma)-\frac{g+3}{8 g} \ell(\Gamma)+\frac{g-1}{4 g} \sum_{e_{i} \in E(\Gamma)} \frac{L_{i} R_{i}}{L_{i}+R_{i}} .
$$

Proof. We obtain the equality by substituting the formula for $\theta(\Gamma)$ given in Proposition 5.13 into the formula for $\varphi(\Gamma)$ given in Theorem 4.3. Since all of $R_{c_{i}, q}, L_{i}, R_{i}, \bar{R}_{c_{j}, \bar{p}_{i}}$, and $\bar{R}_{j}$ are positive, the inequality follows from the equality if $v(q) \geq 3$ for each $q \in V(\Gamma)$.

Arguments similar to those in the proof of Proposition 5.14 can be used to derive another formula for $\lambda(\Gamma)$. Using Proposition 5.13 and Corollary 4.4, we obtain

Proposition 5.15. Let $\Gamma$ be a bridgeless simple pm-graph with genus $g$ and with at least three vertices. Then we have

$$
\begin{aligned}
\lambda(\Gamma)= & \frac{3 g+3}{8 g+4} \tau(\Gamma)+\frac{3 g-1}{16(2 g+1)} \ell(\Gamma)+\frac{g-1}{16 g+8} \sum_{e_{i} \in E(\Gamma)} \frac{L_{i} R_{i}}{L_{i}+R_{i}} \\
& +\frac{1}{8 g+4} \sum_{e_{i} \in E(\Gamma)} \frac{R_{i}}{L_{i}+R_{i}} \sum_{e_{j} \in E\left(\bar{\Gamma}_{i}\right)} \frac{L_{j} \bar{R}_{c_{j}, \bar{p}_{i}}}{L_{j}+\bar{R}_{j}}+\frac{1}{8 g+4} \sum_{q \in V(\Gamma)}(v(q)-3) \sum_{e_{i} \in E(\Gamma)} \frac{L_{i} R_{c_{i}, q}}{L_{i}+R_{i}} .
\end{aligned}
$$

In particular, if $v(q) \geq 3$ for each $q \in V(\Gamma)$, then we have

$$
\lambda(\Gamma) \geq \frac{3 g+3}{8 g+4} \tau(\Gamma)+\frac{3 g-1}{16(2 g+1)} \ell(\Gamma)+\frac{g-1}{16 g+8} \sum_{e_{i} \in E(\Gamma)} \frac{L_{i} R_{i}}{L_{i}+R_{i}} .
$$


Deriving new formulas for $\tau(\Gamma)$ and $\theta(\Gamma)$ helped us obtain additional formulas for $\varphi(\Gamma)$ in Proposition 5.10 and Proposition 5.13. We will show that these formulas lead to improved lower bounds for $\varphi(\Gamma)$. These new lower bounds are much more stronger than the ones given in Theorem 5.1. First, we will need to define the quantities below, which were used in first [C1, Section 3.9] and [C3] to give lower bounds for $\tau(\Gamma)$ and to establish a connection between $\tau(\Gamma)$ and the edge connectivity $\Lambda(\Gamma)$.

In the rest of the paper, for any given pm-graph $\Gamma$ we will use the following notation $x(\Gamma)$ and $y(\Gamma)$, or simply $x$ and $y$ if there is no danger of confusion:

$$
\begin{aligned}
& x(\Gamma)=\sum_{e_{i} \in E(\Gamma)} \frac{L_{i}^{2} R_{i}}{\left(L_{i}+R_{i}\right)^{2}}+\frac{3}{4} \sum_{e_{i} \in E(\Gamma)} \frac{L_{i} R_{i}^{2}}{\left(L_{i}+R_{i}\right)^{2}}-\frac{3}{4} \sum_{e_{i} \in E(\Gamma)} \frac{L_{i}\left(R_{a_{i}, p}-R_{b_{i}, p}\right)^{2}}{\left(L_{i}+R_{i}\right)^{2}}, \\
& y(\Gamma)=\frac{1}{4} \sum_{e_{i} \in E(\Gamma)} \frac{L_{i} R_{i}^{2}}{\left(L_{i}+R_{i}\right)^{2}}+\frac{3}{4} \sum_{e_{i} \in E(\Gamma)} \frac{L_{i}\left(R_{a_{i}, p}-R_{b_{i}, p}\right)^{2}}{\left(L_{i}+R_{i}\right)^{2}} .
\end{aligned}
$$

If $\Gamma-e_{i}$ is not connected for an edge $e_{i}$, i.e. $R_{i}$ is infinite and $\left(R_{a_{i}, p}-R_{b_{i}, p}\right)^{2}=R_{i}^{2}$, the summands should be replaced with their corresponding limits as $R_{i} \longrightarrow \infty$.

By Equation (35) and Proposition 3.5

$$
\tau(\Gamma)=\frac{\ell(\Gamma)}{12}-\frac{x(\Gamma)}{6}+\frac{y(\Gamma)}{6}
$$

and it follows from Equation (35) that

$$
x(\Gamma)+y(\Gamma)=\sum_{e_{i} \in E(\Gamma)} \frac{L_{i} R_{i}}{L_{i}+R_{i}} .
$$

Next, we will express the bounds for $\varphi(\Gamma)$ found above in terms of $x(\Gamma)$ and $y(\Gamma)$.

Proposition 5.16. Let $\Gamma$ be a bridgeless simple pm-graph with genus $g$ and with at least three vertices. Then we have the following two inequalities:

If $v(p) \geq 4$ for each $p \in V(\Gamma)$,

If $v(p) \geq 3$ for each $p \in V(\Gamma)$,

$$
\varphi(\Gamma) \geq \frac{g-1}{6 g} \ell(\Gamma)-\frac{2 g+7}{6 g} x(\Gamma)+\frac{8 g+1}{6 g} y(\Gamma) .
$$

$$
\varphi(\Gamma) \geq \frac{g-1}{6 g} \ell(\Gamma)-\frac{g+2}{3 g} x(\Gamma)+\frac{5 g+1}{6 g} y(\Gamma) .
$$

Proof. By Equation (36) $\tau(\Gamma)=\frac{\ell(\Gamma)}{12}-\frac{x(\Gamma)}{6}+\frac{y(\Gamma)}{6}$, and by Equation (37) $x(\Gamma)+y(\Gamma)=$ $\sum_{e_{i} \in E(\Gamma)} \frac{L_{i} R_{i}}{L_{i}+R_{i}}$. Substituting these into the inequalities given in Propositions 5.11 and 5.14 gives the results.

Let $\Gamma$ be a metrized graph such that $v(p)=n \geq 2$ for each $p \in V(\Gamma)$. We call such a $\Gamma$ an $n$-regular metrized graph, and we extend the definition to pm-graphs.

We consider a specific case in the following theorem.

Theorem 5.17. Let $\Gamma$ be a bridgeless simple pm-graph with $\#(V(\Gamma))=v \geq 3$. Suppose $\Gamma$ is $n$-regular and each edge in $E(\Gamma)$ has the same length. If $n \geq 4$, we have

$$
\frac{\varphi(\Gamma)}{\ell(\Gamma)} \geq \frac{v^{4}\left(n^{2}-4 n+10\right)(n-2)+4 v^{3}\left(n^{2}-n-11\right)-v^{2}(74 n-364)+12 v(5 n-43)+216}{6 n^{2} v^{3}(v(n-2)+2)} .
$$




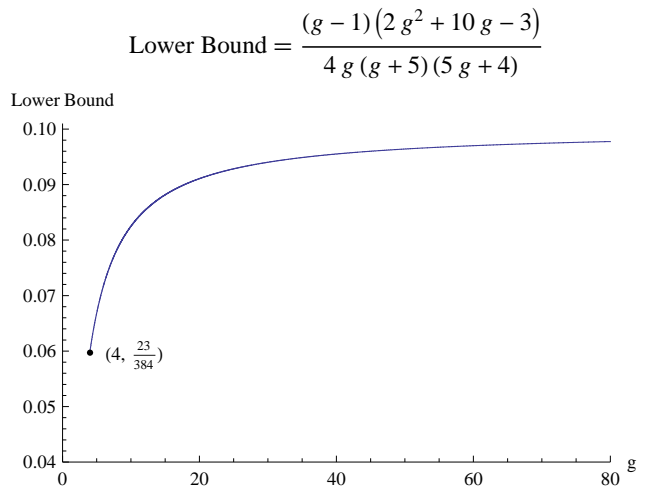

FiguRe 7. Lower bound to $\varphi(\Gamma)$ for $\Gamma$ as in Theorem 5.18 ,

If $n=3$, we have

$$
\varphi(\Gamma) \geq \frac{4 v^{4}-8 v^{3}+91 v^{2}-222 v+144}{54 v^{3}(v+2)} \ell(\Gamma) .
$$

Proof. Let $\#(E(\Gamma))=e$. By basic combinatorial graph theory, we have $e=\frac{n \cdot v}{2}$ for a $n$ regular metrized graph. To make the proof simpler, we can assume that $\ell(\Gamma)=1$ by using Remark 4.8. Since the edge lengths are equal, $L_{i}=\frac{1}{e}$ for each edge $e_{i} \in E(\Gamma)$. Then $x+y=\sum_{e_{i} \in E(\Gamma)} \frac{L_{i} R_{i}}{L_{i}+R_{i}}=\frac{1}{e} \sum_{e_{i} \in E(\Gamma)} \frac{R_{i}}{L_{i}+R_{i}}=\frac{(v-1)}{e}$ by Equation (91). Then the result follows from the inequalities in Proposition 5.16 and the inequality $y \geq \frac{v+6}{4 v}(x+y)^{2}$ (see the article [C3, Theorem 6.9 part (3)]).

When $n=3$ and $v=4$, we have $\varphi(\Gamma)=\frac{17}{288} \ell(\Gamma)$ for a simple pm-graph as in Theorem 5.17 . Therefore, the inequalities given in Theorem 5.17 are sharp.

By using relations between $x(\Gamma), y(\Gamma), \tau(\Gamma)$ and $\Lambda(\Gamma)$, we will derive Theorem 5.18 and Theorem 5.19 which are the two main results on $\varphi(\Gamma)$ :

Theorem 5.18. Let $\Gamma$ be a bridgeless simple pm-graph with genus $g$ and with $\#(V(\Gamma))=$ $v \geq 3$. Suppose $v(p) \geq 4$ for each $p \in V(\Gamma)$. Then we have the following inequalities

$$
\varphi(\Gamma) \geq \frac{2 g^{2}(v+10)-2 g(5 v+2)-19 v-16}{4 g(5 g+4)(v+6)} \ell(\Gamma) .
$$

In particular, we have (see Figure 7),

$$
\varphi(\Gamma) \geq \frac{\left(2 g^{2}+10 g-3\right)(g-1)}{4 g(g+5)(5 g+4)} \ell(\Gamma) .
$$

Moreover, if $\Lambda(\Gamma) \geq \frac{4(5 g+4)}{2 g+7}$, the bounds above can be improved to

$$
\varphi(\Gamma) \geq\left(\frac{(g-1) \Lambda(\Gamma)^{2}(v+6)-4 v(2 g+7) \Lambda(\Gamma)+8 v(5 g+4)}{6 g(v+6) \Lambda(\Gamma)^{2}}\right) \ell(\Gamma)
$$

and

$$
\varphi(\Gamma) \geq\left(\frac{g-1}{6 g}\left(1-\frac{4}{\Lambda(\Gamma)}\right)^{2}+\frac{2(g-1)(\Lambda(\Gamma)+2 g-4)}{g(g+5) \Lambda(\Gamma)^{2}}\right) \ell(\Gamma) .
$$




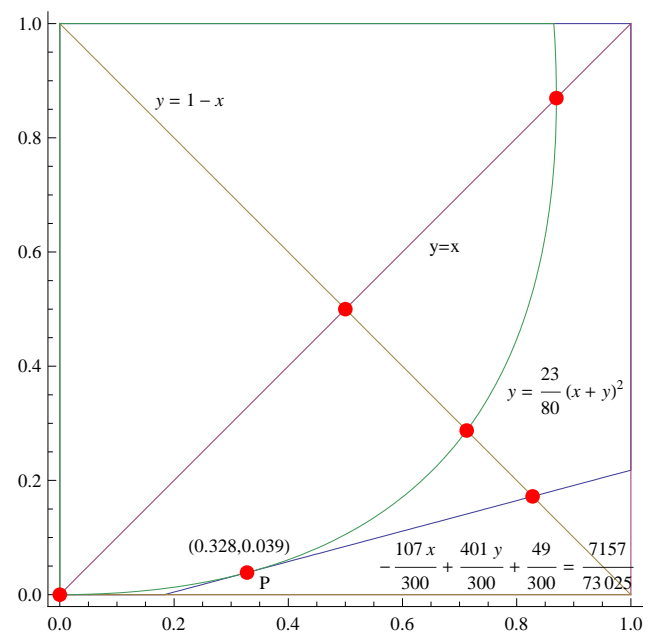

Figure 8. Relations between $x$ and $y$, and the lower bound to $\varphi(\Gamma)$, when $\Gamma$ is as in Theorem 5.18 and $\Lambda(\Gamma)=2, g=50$, and $v=40$.

Proof. To make the proof simpler, we can assume that $\ell(\Gamma)=1$ by using Remark 4.8, By Proposition 5.16 for our case, we have

$$
\varphi(\Gamma) \geq \frac{g-1}{6 g} \ell(\Gamma)-\frac{2 g+7}{6 g} x+\frac{8 g+1}{6 g} y .
$$

On the other hand, by [C3, Theorem 6.9], we have $1 \geq x+y, x \geq 0, y \geq 0, x \geq$ $(\Lambda(\Gamma)-1) y$ and $y \geq \frac{v+6}{4 v}(x+y)^{2}$. Therefore, it will be enough to find $c=c(g, v)$ such that $\frac{g-1}{6 g}-\frac{2 g+7}{6 g} x+\frac{8 g+1}{6 g} y \geq c$ for any given fixed $g$ and $v$. We can choose $c$ such that $\frac{g-1}{6 g}-\frac{2 g+7}{6 g} x+\frac{8 g+1}{6 g} y=c$ is the tangent to the parabola $y=\frac{v+6}{4 v}(x+y)^{2}$. By elementary calculus, the tangency point will be given by $x_{0}=\frac{9 v\left(4 g^{2}+16 g+7\right)}{4(v+6)(5 g+4)^{2}}$ and $y_{0}=\frac{v(2 g+7)^{2}}{4(v+6)(5 g+4)^{2}}$. These give $c=c(g, v)=\frac{2 g^{2}(v+10)-2 g(5 v+2)-19 v-16}{4 g(5 g+4)(v+6)}$. This proves the first inequality in the theorem. An example is shown in Figure 8 .

By expressing $c(g, v)$ as a rational function in $v$, with rational coefficients involving $g$, we can rewrite $c(g, v)$ as $\frac{2 g^{2}-10 g-19}{4 g(5 g+4)}+\frac{8 g^{2}+56 g+98}{4 g(5 g+4)} \frac{1}{v+6}$. This is decreasing as a function of $v$ for each fixed $g$. Since $v(p) \geq 4$ for each $p \in V(\Gamma)$, we have $g=e-v+1 \geq \frac{4 v}{2}-v+1=v+1$. Thus the second inequality follows by substituting $v=g-1$ into the first inequality.

Whenever $\Lambda(\Gamma) \geq \frac{4(5 g+4)}{2 g+7}$, we can choose $c(g, v)$ such that $\frac{g-1}{6 g}-\frac{2 g+7}{6 g} x+\frac{8 g+1}{6 g} y=c(g, v)$ passes through the point of intersection of $x=(\Lambda(\Gamma)-1) y$ and $y=\frac{v+6}{4 v}(x+y)^{2}$. The final two results follow from this by elementary calculus.

Theorem 5.19. Let $\Gamma$ be a bridgeless simple pm-graph with genus $g$ and with $\#(V(\Gamma))=$ $v \geq 3$. Then we have the following inequalities.

If $v(p) \geq 3$ for each $p \in V(\Gamma)$, then we have

$$
\varphi(\Gamma) \geq \frac{g^{2}(v+14)-2 g(3 v+2)-7 v-10}{2 g(7 g+5)(v+6)} \ell(\Gamma) .
$$




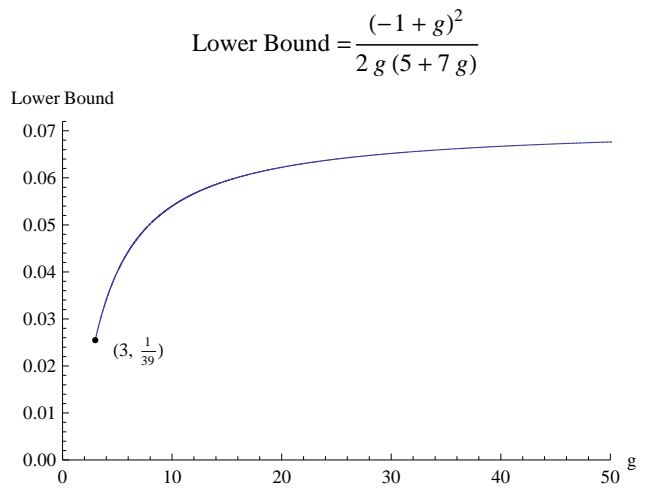

Figure 9. Lower bound to $\varphi(\Gamma)$ for $\Gamma$ as in Theorem 5.19.

In particular, since $2(g-1) \geq v$ we have (see Figure 9),

$$
\varphi(\Gamma) \geq \frac{(g-1)^{2}}{2 g(7 g+5)} \ell(\Gamma)
$$

Moreover, if $\Lambda(\Gamma) \geq \frac{7 g+5}{g+2}$, the bounds above can be improved to

$$
\varphi(\Gamma) \geq\left(\frac{(g-1)(v+6) \Lambda(\Gamma)^{2}-8 v(g+2) \Lambda(\Gamma)+4 v(7 g+5)}{6 g(v+6) \Lambda(\Gamma)^{2}}\right) \ell(\Gamma)
$$

and

$$
\varphi(\Gamma) \geq\left(\frac{g-1}{6 g}\left(1-\frac{4}{\Lambda(\Gamma)}\right)^{2}+\frac{2(g-1)^{2}}{g(g+2) \Lambda(\Gamma)^{2}}\right) \ell(\Gamma) .
$$

Proof. The proof follows from arguments similar to those in the proof of Theorem 5.18. Note that we can use the second inequality in Proposition 5.16 for this time. For the reader's convenience, we give some of the details.

We can assume that $\ell(\Gamma)=1$ by using Remark 4.8. By Proposition 5.16 for this case, we have

$$
\varphi(\Gamma) \geq \frac{g-1}{6 g} \ell(\Gamma)-\frac{g+2}{3 g} x+\frac{5 g+1}{6 g} y .
$$

On the other hand, by [C3, Theorem 6.9], we have $1 \geq x+y, x \geq 0, y \geq 0, x \geq(\Lambda(\Gamma)-$ 1) $y$ and $y \geq \frac{v+6}{4 v}(x+y)^{2}$. Therefore, it will be enough to find $c(g, v)$ such that $\frac{g-1}{6 g}-$ $\frac{g+2}{6 g} x+\frac{5 g+1}{6 g} y \geq c(g, v)$ for any given fixed $g$ and $v$. We can choose $c=c(g, v)$ such that $\frac{g-1}{6 g}-\frac{g+2}{3 g} x+\frac{5 g+1}{6 g} y=c$ is the tangent to the parabola $y=\frac{v+6}{4 v}(x+y)^{2}$. By elementary calculus, the tangency point will be given by $x=\frac{12 v\left(2 g^{2}+5 g+2\right)}{(v+6)(7 g+5)^{2}}$ and $y=\frac{4 v(g+2)^{2}}{(v+6)(7 g+5)^{2}}$. These give $c(g, v)=\frac{g^{2}(v+14)-2 g(3 v+2)-7 v-10}{2 g(7 g+5)(v+6)}$. This proves the first inequality in the theorem.

By expressing $c(g, v)$ as a rational function in $v$, with rational coefficients involving $g$, we can rewrite $c(g, v)$ as $\frac{g^{2}-6 g-7}{2 g(7 g+5)}+\frac{4\left(g^{2}+4 g+4\right)}{g(7 g+5)} \frac{1}{v+6}$. This is decreasing as a function of $v$ for each fixed $g$. Since $v(p) \geq 3$ for each $p \in V(\Gamma), 2 e=\sum_{p \in V(\Gamma)} v(p) \geq 3 v$. Therefore, $g=e-v+1 \geq \frac{3 v}{2}-v+1=\frac{v}{2}+1$. Thus, the second inequality follows by substituting $v=2(g-1)$ into the first inequality. 
Whenever $\Lambda(\Gamma) \geq \frac{(7 g+5)}{g+2}$, we can choose $c(g, v)$ such that $\frac{g-1}{6 g}-\frac{g+2}{3 g} x+\frac{5 g+1}{6 g} y=c(g, v)$ passes through the point of intersection of $x=(\Lambda(\Gamma)-1) y$ and $y=\frac{v+6}{4 v}(x+y)^{2}$. The final two results follow from this by elementary calculus.

Remark 5.20. For any given simple bridgeless pm-graph $\Gamma$ with genus $g \geq 2$ and a vertex set $V(\Gamma)$, we can always find a non-empty vertex subset $V(\Gamma)^{\prime}:=\{p \in V(\Gamma) \mid v(p) \geq 3\}$ by removing vertices of valence 2 if there is any. By valence property of $\varphi(\Gamma)$ (Remark 4.7), this does not change $\varphi(\Gamma)$. We call $V(\Gamma)^{\prime}$ be the minimal vertex set of $\Gamma$. Note that $g \geq \frac{v}{2}+1$, where $\#\left(V(\Gamma)^{\prime}\right)=v$.

Theorem 5.21. Let $\Gamma$ be an irreducible pm-graph of genus $\bar{g} \geq 2$. Then we have $\varphi(\Gamma) \geq$ $t(\bar{g}) \cdot \ell(\Gamma)$, where $t(2)=\frac{1}{27}, t(3)=\frac{1}{30}$, and $t(\bar{g})=\frac{(\bar{g}-1)^{2}}{2 \bar{g}(7 \bar{g}+5)}$ for $\bar{g} \geq 4$.

Proof. This summarizes the best results we have shown above.

First, by considering Proposition 4.17 or equivalently [Fa, Lemma 5.14], it will be enough to prove the desired lower bound inequalities for irreducible simple pm-graphs. Since every irreducible pm-graph is bridgeless, proving these lower bounds for bridgeless simple pmgraphs will implies that these lower bounds hold for irreducible pm-graphs.

Let $\Gamma$ be a bridgeless simple pm-graph. We can work with a minimal vertex set $V(\Gamma)$ by Remark 5.20, Let $\#(V(\Gamma))=v$.

If $v=1$, then $\Gamma$ is a bouquet graph. By Proposition 5.3, we have $\varphi(\Gamma)=\frac{g-1}{6 g} \ell(\Gamma)$, which is stronger than the desired results.

If $v=2$, then $\Gamma$ is a banana graph. By Proposition 5.4, we have $\varphi(\Gamma) \geq \frac{g(g-1)}{6(g+1)^{2}} \ell(\Gamma)$, which is stronger than what we wanted. When $g=2$, this gives $t(2)=\frac{1}{27}$.

If $v \geq 3$, we have $g \geq 3$ because we work with a minimal vertex set for $\Gamma$ (see Remark 5.20). If $g=3$, we have $\varphi(\Gamma) \geq \frac{1}{30} \ell(\Gamma)$ by Corollary 5.5. If $g \geq 4$, we have $\varphi(\Gamma) \geq \frac{(\bar{g}-1)^{2}}{2 \bar{g}(7 \bar{g}+5)} \ell(\Gamma)$ by Theorem 5.19. These finish the proof of the theorem.

Remark 5.22. By Lemma 4.10, Theorem 5.21 gives another proof of the second inequality in Conjecture [2.8, and improves the lower bounds given in Theorem 5.1. In \$7, we will consider $\bar{g}=3$ case in more detail; and improve the lower bound $t(3)$. Namely, we can take $t(3)=\frac{892-11 \sqrt{79}}{14580} \approx 0.054473927$ by Theorem 7.8 .

In Proposition 5.15, we found an inequality for $\lambda(\Gamma)$ where $\Gamma$ is a simple pm-graph with more than 3 vertices. Using Equation (36) and Equation (37), this inequality can be stated in terms of $x(\Gamma)$ and $y(\Gamma)$ as follows:

$$
\lambda(\Gamma) \geq \frac{g}{8 g+4}+\frac{g y(\Gamma)-x(\Gamma)}{8 g+4}, \quad \text { if } v(p) \geq 3 \text { for each } p \in V(\Gamma) .
$$

Similarly, it can be shown by using Proposition 5.12 and Equations (35), (36), and (37) that

$$
\lambda(\Gamma) \geq \frac{g}{8 g+4}+\frac{g y(\Gamma)-x(\Gamma)}{4 g+2}, \quad \text { if } v(p) \geq 4 \text { for each } p \in V(\Gamma) .
$$

Note that by [C3, Theorem 6.9 part (4)] we have $g y \geq x \geq(\Lambda(\Gamma)-1) y>0$, and recall that $y \geq \frac{v+6}{4 v}(x+y)^{2}$, and $x+y<1$ for any bridgeless metrized graph $\Gamma$ with $\ell(\Gamma)=1$. In general, $y$ can be arbitrarily small. If all edge lengths are equal, then $x+y=\frac{v-1}{e} \ell(\Gamma)$. Any "proper" improvement of these relations between $x$ and $y$ will result in improved lower 


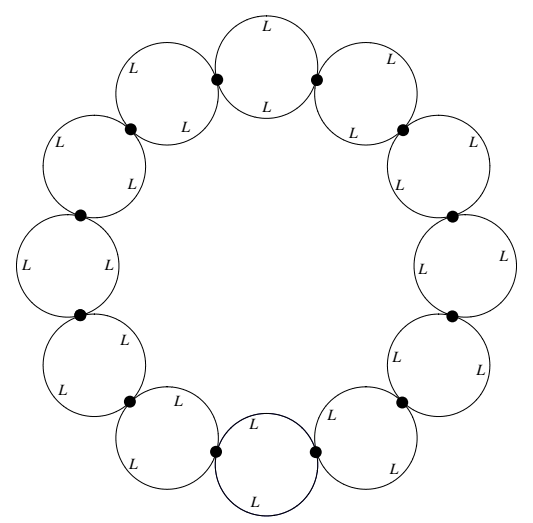

FiguRE 10. The graph $C_{12,2}$.

bounds for $\tau(\Gamma), \lambda(\Gamma)$ and $\varphi(\Gamma)$. For various specific pm-graphs, it is easy to find the exact relation between $x$ and $y$.

We now give an example of a family of pm-graphs for which we can compute $\tau(\Gamma), \lambda(\Gamma)$ and $\varphi(\Gamma)$ by explicit formulas. This example shows how large $\lambda(\Gamma)$ and $\varphi(\Gamma)$ can be. Let $C_{v, n}$ be the metrized graph obtained from the circle graph with $v$ vertices by replacing each of its edges by $n$ multiple edges of equal lengths so that the length of each edge in $E\left(C_{v, n}\right)$ will be $\frac{\ell\left(C_{v, n}\right)}{n \cdot v}$. Figure 10illustrates an example. When $v=1$ and $v=2$, we gave formulas for $\lambda\left(C_{v, n}\right)$ in Corollary 4.14, and for $\varphi\left(C_{v, n}\right)$ in Proposition 5.3 and Proposition 5.4. Suppose $v \geq 3$. Let $V\left(C_{v, n}\right)=\left\{p_{1}, p_{2}, \ldots, p_{v}\right\}$, and $r(x, y)$ be the resistance function on $C_{v, n}$. We have $v(p)=2 n$ for each $p \in V\left(\left(C_{v, n}\right)\right.$, so $e=n \cdot v$, and $g=(n-1) v+1$. Suppose all the edge lengths are equal. Then it follows from parallel and series circuit reductions that $r\left(p_{v}, p_{i}\right)=\frac{i(v-i)}{n^{2} v^{2}} \ell\left(C_{v, n}\right)$. Thus, $\sum_{i=1}^{v-1} r\left(p_{v}, p_{i}\right)=\frac{v^{2}-1}{6 v n^{2}} \ell\left(C_{v, n}\right)$. Moreover, by the symmetry of the graph, we have $\theta(\Gamma)=\sum_{i=1}^{v} \sum_{j=1}^{v}\left(v\left(p_{i}\right)-2\right)\left(v\left(p_{j}\right)-2\right) r\left(p_{i}, p_{j}\right)=\frac{2(n-1)^{2}\left(v^{2}-1\right)}{3 n^{2}} \ell\left(C_{v, n}\right)$. On the other hand, $\tau\left(C_{v, n}\right)=\left(\frac{(n-1)^{2}+1}{12 n^{2}}+\frac{n-1}{6 v n^{2}}\right) \ell(\Gamma)$ by [C2, Example 3.9]. Hence, Corollary 4.4 and these results yield the following proposition:

Proposition 5.23. Let $C_{v, n}$ be a simple pm-graph with equal edge lengths, as defined before. For $v \geq 3$ we have

$$
\lambda\left(C_{v, n}\right)=\frac{v^{2}(n-1)^{2}+3 v(n-1)\left(n^{2}-n+1\right)+5 n^{2}-4 n+2}{12 n^{2}(2(n-1) v+3)} \ell\left(C_{v, n}\right) .
$$

Using the above results along with Theorem 4.3, we obtain the following result.

Proposition 5.24. Let $C_{v, n}$ be a simple pm-graph with equal edge lengths, as defined before. For $v \geq 3$ we have

$$
\varphi\left(C_{v, n}\right)=\frac{(n-1)\left((v-2)^{2}+n v-1\right)}{6 n^{2} v} \ell\left(C_{v, n}\right) .
$$

Note that for a fixed $v, \lim _{n \rightarrow \infty} \varphi\left(C_{v, n}\right)=\frac{1}{6}$, where length of the pm-graph $C_{v, n}$ is kept fixed as 1 . 

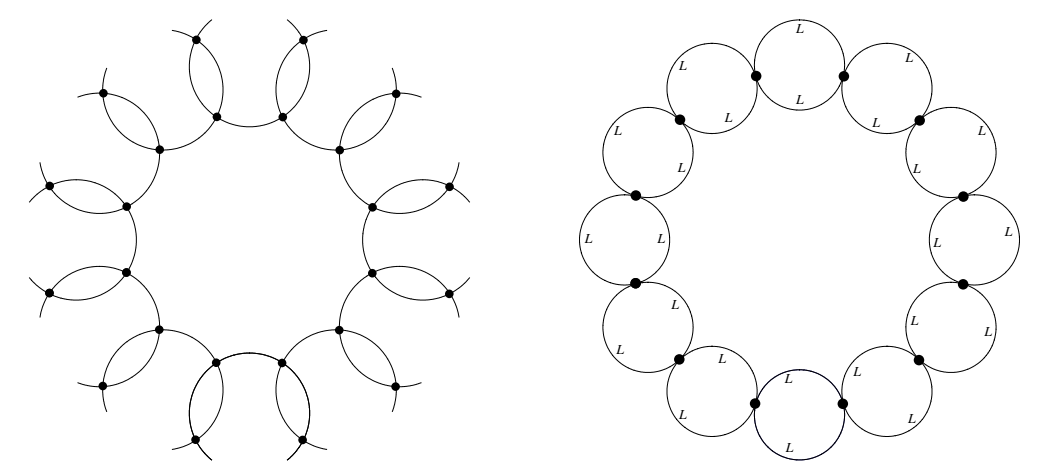

FiguRE 11. A curve with irreducible components and its dual graph.

Corollary 5.25. Let $C_{v, n}$ be a simple pm-graph with equal edge lengths, as defined before. For $v \geq 3$ we have

$$
\begin{aligned}
& \varphi\left(C_{v, 2}\right)=\frac{(v-1)^{2}+2}{24 v} \ell\left(C_{v, 2}\right)=\frac{(g-2)^{2}+2}{24(g-1)} \ell\left(C_{v, 2}\right), \\
& \lambda\left(C_{v, 2}\right)=\frac{(v+2)(v+7)}{48(2 v+3)} \ell\left(C_{v, 2}\right)=\frac{(g+6)(g+1)}{48(2 g+1)} \ell\left(C_{v, 2}\right) .
\end{aligned}
$$

Note that Corollary 5.25 shows that $\varphi(\Gamma)$ and $\lambda(\Gamma)$ can be arbitrarily large for a graph of total length 1.

Next, we will consider the simple complete pm-graphs with arbitrary length distributions, unlike Propositions (5.7) and (5.8). Note that we have $g=\frac{(v-1)(v-2)}{2}$ for a simple complete pm-graph.

Proposition 5.26. Let $\Gamma$ be a simple complete pm-graph on $v$ vertices, where $v>3$. Then we have $\theta(\Gamma)=2(v-3)^{2}(x(\Gamma)+y(\Gamma)), \varphi(\Gamma)=\frac{v(v-3) \ell(\Gamma)}{6(v-1)(v-2)}+\frac{v^{2}-21 v+48}{6(v-1)(v-2)} x(\Gamma)+\frac{11 v^{2}-51 v+60}{6(v-1)(v-2)} y(\Gamma)$ and $\lambda(\Gamma)=\frac{(v-1)^{2}(v-2)^{2}-2}{8\left((v-1)^{2}(v-2)^{2}-1\right)} \ell(\Gamma)+\frac{(v-2)(v-3)\left(v^{2}-7 v+3\right)}{8\left((v-1)^{2}(v-2)^{2}-1\right)} x(\Gamma)+\frac{(v-3)\left(3 v^{3}-15 v^{2}+23 v-6\right)}{8\left((v-1)^{2}(v-2)^{2}-1\right)} y(\Gamma)$.

Proof. We have $v(p)=v-1$ for each $p \in V(\Gamma)$. On the other hand, $r\left(p_{i}, q_{i}\right)=\frac{L_{i} R_{i}}{L_{i}+R_{i}}$ if $p_{i}$ and $q_{i}$ are the end points of the edge $e_{i} \in E(\Gamma)$. Therefore, $\theta(\Gamma)=2(v-3)^{2} \sum_{e_{i} \in E(\Gamma)} \frac{L_{i} R_{i}}{L_{i}+R_{i}}$. Then the formula for $\theta(\Gamma)$ follows from Equation (37).

The formula for $\varphi(\Gamma)$ follows from Equation (36) , the formula for $\theta(\Gamma)$ and Theorem 4.3 . Similarly, the formula for $\theta(\Gamma)$ follows from Equation (36), the formula for $\theta(\Gamma)$ and Corollary 4.4.

\section{Calculations of PM-GRaph invariants using the Discrete Laplacian}

In this section, for a given pm-graph $\Gamma$ we express $\tau(\Gamma)$ and $\theta(\Gamma)$ in terms of the corresponding discrete Laplacian. This leads to fast computer algorithms that can be used to compute $\varphi(\Gamma), \epsilon(\Gamma), \lambda(\Gamma)$ (see the Mathematica codes given at the end). Also, this gives another approach, as in Theorem 6.4, to find lower bounds to these invariants.

The discrete Laplacian matrix of $\mathrm{L}$ of a pm-graph $\Gamma$ is the same as the discrete Laplacian matrix of $\Gamma$ considered only with its metrized graph structure. This is no different than the construction of discrete Laplacian for a weighted graph. The details of its definition will be given below. 
To have a well-defined discrete Laplacian matrix $\mathrm{L}$ for a given pm-graph $\Gamma$, we first choose a vertex set $V(\Gamma)$ for $\Gamma$ in such a way that there are no self-loops, and no multiple edges connecting any two vertices. This can be done for any pm-graph $\Gamma$ by enlarging the vertex set by inserting, whenever needed, additional valence two vertices with q value 0 . Note that enlarging the vertex set in this way does not change the value of $\varphi(\Gamma)$ (or $\epsilon(\Gamma), a(\Gamma)$, or $\lambda(\Gamma)$ ) by Remark 4.7. Such a vertex set $V(\Gamma)$, which can be used in the construction of a discrete Laplacian for $\Gamma$, will be called an optimal vertex set. If two distinct vertices $p$ and $q$ are the end points of an edge, we call them adjacent vertices.

Definition. Let $\Gamma$ be a pm-graph with $e$ edges and with an optimal vertex set $V(\Gamma)$ containing $v$ vertices. Fix an ordering of the vertices in $V(\Gamma)$. Let $\left\{L_{1}, L_{2}, \cdots, L_{e}\right\}$ be a labeling of the edge lengths. The $v \times v$ matrix $\mathrm{A}=\left(a_{p q}\right)$ given by

$$
a_{p q}=\left\{\begin{array}{ll}
0 & \text { if } p=q, \text { or } p \text { and } q \text { are not adjacent. } \\
\frac{1}{L_{k}} & \text { if } p \neq q, \text { and } p \text { and } q \text { are connected by an edge of length } L_{k}
\end{array} .\right.
$$

is called the adjacency matrix of $\Gamma$. Let $\mathrm{D}=\operatorname{diag}\left(d_{p p}\right)$ be the $v \times v$ diagonal matrix given by $d_{p p}=\sum_{s \in V(\Gamma)} a_{p s}$. Then $\mathrm{L}:=\mathrm{D}-\mathrm{A}$ will be called the discrete Laplacian matrix of $\Gamma$. That is, $\mathrm{L}=\left(l_{p q}\right)$ where

$$
l_{p q}= \begin{cases}0 & \text { if } p \neq q, \text { and } p \text { and } q \text { are not adjacent. } \\ -\frac{1}{L_{k}} & \text { if } p \neq q, \text { and } p \text { and } q \text { are connected by an edge of length } L_{k} . \\ -\sum_{s \in V(\Gamma)-\{p\}} l_{p s} & \text { if } p=q\end{cases}
$$

We will denote the pseudo inverse of $\mathrm{L}$ by $\mathrm{L}^{+}$. For properties of $\mathrm{L}$ and $\mathrm{L}^{+}$, see the article [C4] and the references given therein.

Lemma 6.1. [RB2] [RB1] Let $\Gamma$ be a metrized graph with the discrete Laplacian $\mathrm{L}$ and the resistance function $r(x, y)$. For the pseudo inverse $\mathrm{L}^{+}$we have

$$
r(p, q)=l_{p p}^{+}-2 l_{p q}^{+}+l_{q q}^{+}, \quad \text { for any } p, q \in V(\Gamma) .
$$

Let $\Gamma$ be a pm-graph with an optimal vertex set $V(\Gamma)$. Using Lemma 6.1, we can express $\theta(\Gamma)$ in terms of entries of $\mathrm{L}^{+}$as follows:

$$
\theta(\Gamma)=\sum_{p, q \in V(\Gamma)}(v(p)-2+2 \mathbf{q}(p))(v(q)-2+2 \mathbf{q}(q))\left(l_{p p}^{+}-2 l_{p q}^{+}+l_{q q}^{+}\right) .
$$

In particular, suppose that $v(p)-2+2 \mathbf{q}(p)=k$ for each $p \in V(\Gamma)$. Since $2 \bar{g}-2=$ $\sum_{p \in V(\Gamma)}(v(p)-2+2 \mathbf{q}(p)), k=\frac{2 \bar{g}-2}{v}$. On the other hand, we have $\sum_{p, q \in V(\Gamma)}\left(l_{p p}^{+}-2 l_{p q}^{+}+l_{q q}^{+}\right)=$ $2 v \cdot \operatorname{trace}\left(\mathrm{L}^{+}\right)$. This implies that

$$
\theta(\Gamma)=\frac{8(\bar{g}-1)^{2}}{v} \operatorname{trace}\left(\mathrm{L}^{+}\right) .
$$

Theorem 6.2. [C4, Theorem 4.10] Let $\mathrm{L}$ be the $v \times v$ discrete Laplacian matrix for a graph $\Gamma$ with $v$ vertices. Let $p_{i}$ and $q_{i}$ be end points of edge $e_{i}$ for each $i=1,2, \cdots, e$. Then

$$
\tau(\Gamma)=-\frac{1}{12} \sum_{e_{i} \in E(\Gamma)} l_{p_{i} q_{i}}\left(\frac{1}{l_{p_{i} q_{i}}}+l_{p_{i} p_{i}}^{+}-2 l_{p_{i} q_{i}}^{+}+l_{q_{i} q_{i}}^{+}\right)^{2}-\frac{1}{4} \sum_{e_{i} \in E(\Gamma)} l_{p_{i} q_{i}}\left(l_{p_{i} p_{i}}^{+}-l_{q_{i} q_{i}}^{+}\right)^{2}+\frac{1}{v} \operatorname{tr}\left(\mathrm{L}^{+}\right) .
$$


Remark 6.3. For any given pm-graph $\Gamma$, we can choose an optimal vertex set $V(\Gamma)$. Then $\varphi(\Gamma)$ can be computed via a computer algorithm utilizing Theorem 4.3, Equation (40) and Theorem 6.2.

Since a pm-graph is connected, its discrete Laplacian matrix $\mathrm{L}$ of size $v \times v$ has $v-1$ non-zero eigenvalues. Likewise, its pseudo inverse $\mathrm{L}^{+}$has $v-1$ non-zero eigenvalues which are the reciprocal of the nonzero eigenvalues of L. Thus by applying Arithmetic-Harmonic Mean inequality, we have

$$
\operatorname{trace}\left(\mathrm{L}^{+}\right) \geq \frac{(v-1)^{2}}{\operatorname{trace}(\mathrm{L})}
$$

For an $n$-regular metrized graph with equal edge lengths and with total length 1 , we have the following equality for the corresponding discrete Laplacian:

$$
\operatorname{trace}(\mathrm{L})=v \cdot e \cdot n=\frac{v^{2} \cdot n^{2}}{2}
$$

and by [C2, Proof of Theorem 2.24]

$$
\sum_{e_{i} \in E(\Gamma)} \frac{L_{i}^{3}}{\left(L_{i}+R_{i}\right)^{2}} \geq\left(\frac{g}{e}\right)^{2} .
$$

We combine Equation (43) and Equation (42) to obtain

$$
\operatorname{trace}\left(\mathrm{L}^{+}\right) \geq \frac{2}{n^{2}}\left(\frac{v-1}{v}\right)^{2}
$$

Therefore, by using Theorem 6.2, the inequalities (45) and (44), Theorem 4.3, and Remark 4.8, we obtain the following inequality for $\varphi(\Gamma)$ :

Theorem 6.4. Let $(\Gamma, \boldsymbol{q})$ be a pm-graph such that $\boldsymbol{q}(p)$ is constant for each $p \in V(\Gamma)$. Suppose that $\Gamma$ is a n-regular metrized graph with equal edge lengths. Then we have

$$
\varphi(\Gamma) \geq \frac{v^{3}\left(n^{2}+2 n-14\right)-v^{2}(16 n-68)+6 v(2 n-15)+36}{6 n^{2} v^{3}} \ell(\Gamma) .
$$

In particular, if $n=3$, we have

$$
\varphi(\Gamma) \geq \frac{v^{3}+20 v^{2}-54 v+36}{54 v^{3}} \ell(\Gamma) .
$$

Note that when $v=4$ and $n=3, \varphi(\Gamma)=\frac{17}{288}$ for a simple graph as in Theorem 6.4. Thus the inequalities given in Theorem 6.4 are sharp. Note also that the lower bounds given in Theorem 6.4 are independent of $\mathbf{q}(p)$.

\section{EXPLICIT FORMULAS FOR GENUS 3 CUBIC SIMPLE PM-GRAPHS}

In this section, we consider pm-graphs with genus 3, and improve the lower bounds to $\varphi(\Gamma), \lambda(\Gamma)$ by obtaining explicit formulas that are due to the techniques developed in the previous sections and the articles [C2], C3] and [C4].

Recall that it is enough to find lower bounds for simple pm-graphs to find lower bounds to $\varphi(\Gamma), \lambda(\Gamma)$ (see Remark 4.18). Moreover, Faber [Fa, Lemma 5.15] showed that it is enough to consider irreducible cubic simple pm-graphs to find lower bounds for $\varphi(\Gamma)$. There are two types of bridgeless cubic simple pm-graphs of genus 3 [Fa, Figure 3]. These are $\Gamma$ and $\beta$ as illustrated in Figure 12 . 

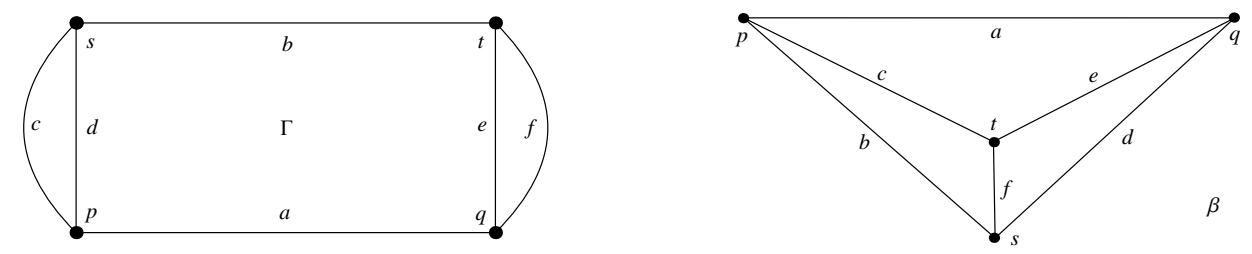

Figure 12. Cubic simple pm-graphs with genus 3.

We will first consider $\Gamma$. We have $V(\Gamma)=\{p, q, t, s\}, E(\Gamma)=\left\{e_{1}, e_{2}, e_{3}, e_{4}, e_{5}, e_{6}\right\}$ and the corresponding edge lengths $\{a, b, c, d, e, f\}$ giving $\ell(\Gamma)=a+b+c+d+e+f$. Let $r_{\Gamma}(x, y)$ be the resistance function in $\Gamma$, and let $M=((c+d)(a+b)+c d)(e+f)+(c+d) e f$. Then by circuit reductions we have

$$
\begin{aligned}
& r_{\Gamma}(p, q)=a((b(c+d)+c d)(e+f)+e f(c+d)) / M, \\
& r_{\Gamma}(p, t)=(b c+b d+c d)(a e+a f+e f) / M, \\
& r_{\Gamma}(p, s)=c d((a+b+f) e+(a+b) f) / M, \\
& r_{\Gamma}(q, t)=((a+b+d) c+(a+b) d) e f / M, \\
& r_{\Gamma}(q, s)=(a(c+d)+c d)(b(e+f)+e f) / M, \\
& r_{\Gamma}(s, t)=b(a(c+d)(e+f)+c d(e+f)+e f(c+d)) / M .
\end{aligned}
$$

By Equation (37), $x(\Gamma)+y(\Gamma)=r_{\Gamma}(p, q)+r_{\Gamma}(s, t)+2\left(r_{\Gamma}(p, s)+r_{\Gamma}(q, t)\right)$. Thus,

$$
x(\Gamma)+y(\Gamma)=(3(a+b)(c d(e+f)+(c+d) e f)+2 a b(c+d)(e+f)+4 c d e f) / M .
$$

Next, we give an explicit formula for the tau constant of $\Gamma$ :

Proposition 7.1. Let $\Gamma$ be the simple pm-graph above. Then

$$
\tau(\Gamma)=\frac{\ell(\Gamma)}{12}-((a+b)((c+f) d e+c f(d+e))+2 c d e f) /(6 M) .
$$

Proof. Recall that $e_{1}$ is an edge of $\Gamma$ with edge length $a$ as described above. Applying [C2, Corollary 5.3] to $\Gamma$ with $e_{1}$, we obtain $\tau(\Gamma)=\tau\left(\Gamma-e_{1}\right)+\frac{a}{12}-\frac{R_{1}}{6}+\frac{A_{p, q, \Gamma-e_{1}}}{a+R_{1}}$. We have $\tau\left(\Gamma-e_{1}\right)=\frac{c+d+e+f}{12}+\frac{b}{4}$ by the additive property of the tau constant [C2, page 11], Corollary 3.6 and [C2, Corollary 2.22]. Moreover, $R_{1}=\frac{c d}{c+d}+b+\frac{e f}{e+f}$ by parallel and series circuit reductions, and $A_{p, q, \Gamma-e_{1}}=\frac{1}{6}\left(\frac{c^{2} d^{2}}{(c+d)^{2}}+\frac{e^{2} f^{2}}{(e+f)^{2}}\right)$ by [C2, Propositions 4.5, 4.6 and 8.9]. This gives the formula for $\tau(\Gamma)$.

Proposition 7.2. Let $\Gamma$ be the simple pm-graph above. Then

$$
\begin{aligned}
& \theta(\Gamma)=(6(a+b)(c d(e+f)+(c+d) e f)+8 a b(c+d)(e+f)+8 c d e f) / M, \\
& \varphi(\Gamma)=\frac{\ell(\Gamma)}{9}-(2(a+b)(c d(e+f)+(c+d) e f)-6 a b(c+d)(e+f)+7 c d e f) /(9 M) .
\end{aligned}
$$

Proof. Since $\theta(\Gamma)=2\left(r_{\Gamma}(p, q)+r_{\Gamma}(p, t)+r_{\Gamma}(p, s)+r_{\Gamma}(q, t)+r_{\Gamma}(q, s)+r_{\Gamma}(s, t)\right)$, we have the formula for $\theta(\Gamma)$. Since $\Gamma$ is simple, $\varphi(\Gamma)=\frac{(5 g-2) \tau(\Gamma)}{g}+\frac{\theta(\Gamma)}{4 g}-\frac{\ell(\Gamma)}{4}$ by Theorem 4.3. Then the result follows from the formula for $\theta(\Gamma)$ and Proposition 7.1 . 
By Equation (36), Proposition 7.1 and Equation (46) we have

$$
\begin{aligned}
& x(\Gamma)=(2(a+b)(c d(e+f)+(c+d) e f)+a b(c+d)(e+f)+3 c d e f) / M, \\
& y(\Gamma)=((a+b)(c d(e+f)+(c+d) e f)+a b(c+d)(e+f)+c d e f) / M .
\end{aligned}
$$

Proposition 7.3. Let $\Gamma$ be the simple pm-graph above. Then we have the following sharp bound $\varphi(\Gamma)>\frac{\ell(\Gamma)}{16}$.

Proof. After doing the algebra we obtain that $\varphi(\Gamma)=\frac{\ell(\Gamma)}{16}+\left(11 / 2(a+b)\left((c-d)^{2} e+(c-\right.\right.$ $\left.d)^{2} f+c(e-f)^{2}+d(e-f)^{2}\right)+7\left(d e(c-f)^{2}+e f(d-c)^{2}+c e(f-d)^{2}+c f(e-d)^{2}+c d(e-f)^{2}+\right.$ $\left.\left.d f(c-e)^{2}\right)\right) /(144 M)+\left(\left(14\left(a^{2}+b^{2}\right)+220 a b\right)(c+d)(e+f)+3(a+b)\left(\left(c^{2}+d^{2}\right)(e+f)+(c+\right.\right.$ $\left.\left.d)\left(e^{2}+f^{2}\right)\right)\right) /(288 M)$. This clearly gives that $\varphi(\Gamma)>\frac{\ell(\Gamma)}{16}$. If $a=b$ and $c=d=e=f$, then we have $\ell(\Gamma)=2 a+4 c$, and $\varphi(\Gamma)=\frac{\ell(\Gamma)}{16}+\frac{a(62 a+3 c)}{72(2 a+c)}$. Since $\Gamma$ has genus 3 , we have $a>0$. Moreover, $\varphi(\Gamma)$ approaches to $\frac{\ell(\Gamma)}{16}$ as $a \rightarrow 0$.

Proposition 7.4. Let $\Gamma$ be the simple pm-graph above. Then we have

$$
\lambda(\Gamma)=\frac{3}{28} \ell(\Gamma)+(4 a b(c+d)(e+f)+(a+b)(c d(e+f)+(c+d) e f)) /(28 M) .
$$

In particular, $\lambda(\Gamma)>\frac{3}{28} \ell(\Gamma)$.

Proof. Since $\Gamma$ is simple, by Corollary 4.4 we have $\lambda(\Gamma)=\frac{(3 g-3) \tau(\Gamma)}{4 g+2}+\frac{\theta(\Gamma)}{16 g+8}+\frac{(g+1) \ell(\Gamma)}{16 g+8}$. Hence, the result follows from Proposition 7.1 and the formula of $\theta(\Gamma)$ given in Proposition 7.2 .

For $a=b$ and $c=d=e=f, \lambda(\Gamma)=\frac{3}{28} \ell(\Gamma)+\frac{a}{14}$. Therefore, $\lambda(\Gamma)$ approaches to $\frac{3}{28} \ell(\Gamma)$ as $a \rightarrow 0$.

Now, we will consider $\beta$. We have $V(\beta)=\{p, q, s, t\}$, and the edge lengths $\{a, b, c, d, e, f\}$ giving $\ell(\beta)=a+b+c+d+e+f$.

$$
\mathrm{L}=\left[\begin{array}{cccc}
\frac{1}{a}+\frac{1}{b}+\frac{1}{c} & -\frac{1}{a} & -\frac{1}{b} & -\frac{1}{c} \\
-\frac{1}{a} & \frac{1}{a}+\frac{1}{d}+\frac{1}{e} & -\frac{1}{d} & -\frac{1}{e} \\
-\frac{1}{b} & -\frac{1}{d} & \frac{1}{b}+\frac{1}{d}+\frac{1}{f} & -\frac{1}{f} \\
-\frac{1}{c} & -\frac{1}{e} & -\frac{1}{f} & \frac{1}{c}+\frac{1}{e}+\frac{1}{f}
\end{array}\right] \text {, and let } \mathrm{J}=\left[\begin{array}{cccc}
1 & 1 & 1 & 1 \\
1 & 1 & 1 & 1 \\
1 & 1 & 1 & 1 \\
1 & 1 & 1 & 1
\end{array}\right] \text {. }
$$

Then, we compute the pseudo inverse $\mathrm{L}^{+}$of $\mathrm{L}$ by using the formula $\mathrm{L}^{+}=\left(\mathrm{L}-\frac{1}{4} \mathrm{~J}\right)^{-1}+\frac{1}{4} \mathrm{~J}$. We have computed this by using Mathematica. Since the entries of $\mathrm{L}^{+}$are quite lengthy, we do not give the formula here. Let $N=a b d+a c d+b c d+a b e+a c e+b c e+b d e+c d e+$ $a b f+a c f+b c f+a d f+c d f+a e f+b e f+d e f$. We obtain the following formula for $\tau(\beta)$ by applying Theorem 6.2 ,

$$
\tau(\beta)=\frac{\ell(\beta)}{12}-(c d e f+b(c d(2 e+f)+(c+d) e f)+a(c d(e+2 f)+(c+d) e f)+a b(c(d+e+
$$
$f)+d e+d f+2 e f)) /(6 N)$.

We have $\theta(\beta)=8$ trace $\left(\mathrm{L}^{+}\right)$by Equation (41), so

$\theta(\beta)=6 c d e f+b(8 c d e+6(c d+c e+d e) f)+a(c(6 d e+(8 d+6 e) f)+6 d e f+b(6 c(d+e+$ $f)+6 d e+(6 d+8 e) f))) / N$.

We have $\theta(\beta)=2(x(\beta)+y(\beta))$ by Equation (41), and $\tau(\beta)=\frac{\ell(\beta)}{12}-\frac{x(\beta)}{6}+\frac{y(\beta)}{6}$ by Equation (36). These give

$y(\beta)=(a b c d+a b c e+a b d e+a c d e+b c d e+a b c f+a b d f+a c d f+b c d f+a b e f+a c e f+$ $b c e f+$ adef + bdef + cdef $) / N$, and $x(\beta)=2 y(\beta)+($ bcde + acdf + abef $) / N$. 


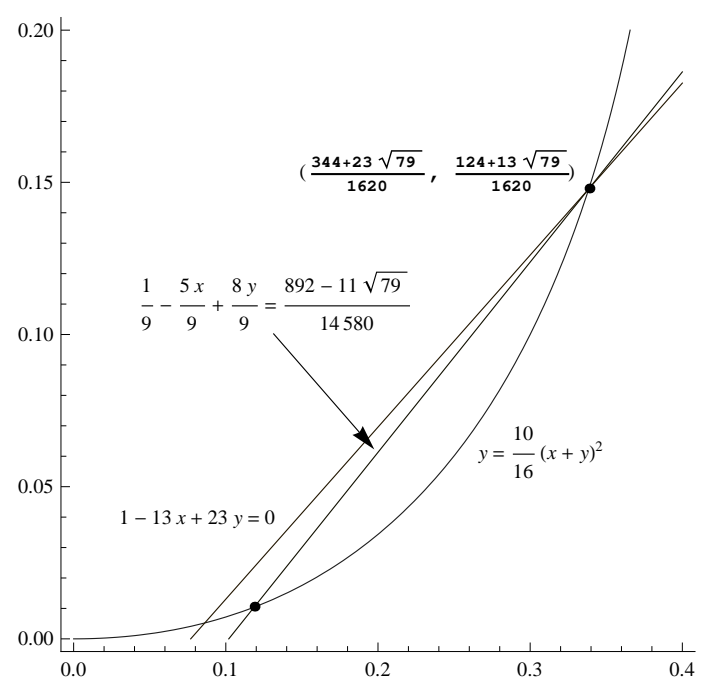

Figure 13. Giving bounds to $\varphi(\beta)$.

By Theorem 4.3 and above formulas for $\tau(\beta)$ and $\theta(\beta)$, we have $\varphi(\beta)=\frac{\ell(\beta)}{9}-\frac{5}{9} x(\beta)+\frac{8}{9} y(\beta)$. Note that this shows that the second lower bound given in Proposition 5.16 is sharp.

Proposition 7.5. Let $\beta$ be the simple pm-graph above. Then $\lambda(\beta)=\frac{3}{28} \ell(\beta)+($ cdef + $b(d e f+c(d f+e f))+a(d e f+c(d e+e f)+b(d e+d f+c(d+e+f)))) /(28 N)$. In particular, $\lambda(\beta)>\frac{3}{28} \ell(\beta)$.

Proof. Since $\beta$ is simple, by Corollary 4.4 we have $\lambda(\beta)=\frac{(3 g-3) \tau(\beta)}{4 g+2}+\frac{\theta(\beta)}{16 g+8}+\frac{(g+1) \ell(\beta)}{16 g+8}$. Then the result follows from the above formulas for $\tau(\beta)$ and $\theta(\beta)$.

For $a=f=\frac{\ell(\beta)}{2}-k$ and $b=c=d=e=\frac{k}{2}, \lambda(\beta)=\frac{3}{28} \ell(\beta)+\frac{k(\ell(\beta)-2 k)}{56(\ell(\beta)-k)^{2}} \ell(\beta)$. Therefore, $\lambda(\beta)$ approaches to $\frac{3}{28} \ell(\beta)$ as $k \rightarrow 0$.

Proposition 7.6. Let $\beta$ be as before. Then $13 x(\beta) \geq \ell(\beta)+23 y(\beta)$.

Proof. Let $h$ be the function given by $h(a, b, c, d)=a^{2} b c+a b^{2} c+a b c^{2}+a^{2} b d+a b^{2} d+a^{2} c d-$ $12 a b c d+b^{2} c d+a c^{2} d+b c^{2} d+a b d^{2}+a c d^{2}+b c d^{2}$. We have Arithmetic-Harmonic mean inequality for any given $a>0, b>0, c>0$ and $d>0$. Namely, $a+b+c+d \geq \frac{16}{\frac{1}{a}+\frac{1}{b}+\frac{1}{c}+\frac{1}{d}}$ with equality iff $a=b=c=d$. Note that this is equivalent to $h(a, b, c, d) \geq 0$. By using the above formulas of $x(\beta)$ and $y(\beta)$, we obtain $\ell(\Gamma)-13 x(\Gamma)+23 y(\Gamma)=(h(b, c, d, e)+h(a, c, d, f)+h(a, b, e, f)+$ $\left.a^{2} b d+a b^{2} d+a b d^{2}+a^{2} c e+a c^{2} e+a c e^{2}+b^{2} c f+b c^{2} f+d^{2} e f+d e^{2} f+b c f^{2}+d e f^{2}\right) / N$. This gives the result.

Proposition 7.7. Let $\beta$ be as before. Then $\varphi(\beta) \geq \frac{892-11 \sqrt{79}}{14580} \ell(\beta) \approx 0.054473927 \ell(\beta)$.

Proof. We assume that $\ell(\beta)=1$. We have $y>\frac{10}{16}(x+y)^{2}$ by [C3, Theorem 6.9 part (3)], and $13 x \geq \ell(\beta)+23 y$ by Proposition 7.6. Note that the parabola $y=\frac{10}{16}(x+y)^{2}$ and the line $13 x=\ell(\beta)+23 y$ have intersection point with coordinates $x_{0}=\frac{344+23 \sqrt{79}}{1620}$ and $y_{0}=\frac{124+13 \sqrt{79}}{1620}$ as also illustrated in Figure 13. These inequalities imply that $\frac{\ell(\beta)}{9}-\frac{5}{9} x+\frac{8}{9} y \geq \frac{892-11 \sqrt{79}}{14580}$. That is, $\varphi(\beta) \geq \frac{892-11 \sqrt{79}}{14580}$. Then the result follows from Remark 4.8. 
When $a=b=c=d=e=f=\frac{1}{6}, \varphi(\beta)=\frac{17}{288}$. There is considerable computational evidence, based on the numerical optimization methods in Mathematica, that the lower bound in Proposition 7.7 can be taken as $\frac{17}{288} \ell(\beta)$, which is also conjectured by Faber [Fa, Remark 5.1].

The lower bounds to $\varphi(\Gamma)$ were given in Theorem 5.21. When $\bar{g}=3$, the previous lower bound can be improved as follows:

Theorem 7.8. Let $\Gamma$ be an irreducible pm-graph with genus $\bar{g}=3$. Then we have $\varphi(\Gamma) \geq$ $\frac{892-11 \sqrt{79}}{14580} \ell(\beta) \approx 0.054473927 \ell(\beta)$.

Proof. First, by considering Proposition 4.17 or equivalently [Fa, Lemma 5.14], it will be enough to prove the given lower bound inequalities for any given irreducible simple pmgraphs. Since every irreducible pm-graph is bridgeless, proving these lower bounds for bridgeless simple pm-graphs will implies that these lower bounds hold for irreducible pm-graphs. Moreover, by using [Fa, Lemma 5.15], it will be enough to consider bridgeless simple cubic pm-graphs of genus 3. Recall that there are only two types of bridgeless cubic pm-graph of genus $g=3$. Hence, the result follows from Propositions (7.7) and (7.3).

One can use the techniques that we have developed to compute $\varphi(\Gamma)$ and $\lambda(\Gamma)$ explicitly for other graphs, similarly to what we did in this section. However, the formulas become too large to include as $g$ gets bigger.

\section{REFERENCES}

[AM1] A. Moriwaki, A sharp slope inequality for general stable fibrations of curves, J. reine angew. Math. 480 (1996), 177-195. MR 97m:14029, http://arxiv.org/abs/alg-geom/9601003

[AM2] A. Moriwaki, Bogomolov conjecture over function fields for stable curves with only irreducible fibers, Comp. Math. 105 (1997), 125-140. CMP 97:10, http://arxiv.org/abs/alg-geom/9505003

[AM3] A. Moriwaki, Bogomolov conjecture for curves of genus 2 over function fields, J. Math. Kyoto Univ., 36 (1996), 687-695. CMP 97:11, http://arxiv.org/abs/alg-geom/9509008

[AM4] A. Moriwaki, Relative Bogomolov's inequality and the cone of positive divisors on the moduli space of stable curves, J. Amer. Math. Soc., 11 (1998), 569-600.

[BB] B. Bollabás, Extremal Graph Theory, Dover Publications INC., Mineola, New York, 2004.

[BF] M. Baker and X. Faber, Metrized graphs, Laplacian operators, and electrical networks, Quantum graphs and their applications, 15-33, Contemp. Math., 415, Amer. Math. Soc., Providence, RI, 2006.

[BR] M. Baker and R. Rumely, Harmonic analysis on metrized graphs, Canadian J. Math: May 9, 2005, http://arxiv.org/abs/math.NT/0407427

[C1] Z. Cinkir, The Tau Constant of Metrized Graphs, Thesis at University of Georgia, 2007.

[C2] Z. Cinkir, The tau constant of a metrized graph and its behavior under graph operations, preprint, http://arxiv.org/abs/0901.0407v3

[C3] Z. Cinkir, The tau constant and the edge connectivity of a metrized graph, preprint, http://arxiv.org/abs/0901.1481v2

[C4] Z. Cinkir, The tau constant and the discrete Laplacian of a metrized graph, preprint, http://arxiv.org/abs/0902.3401v2

[CR] T. Chinburg and R. Rumely, The capacity pairing, J. reine angew. Math. 434 (1993), 1-44.

[Fa] X. W.C. Faber, The geometric bogomolov conjecture for small genus curves, preprint, http://arxiv.org/abs/0803.0855v2

[G] W. Gubler, The Bogomolov conjecture for totally degenerate abelian varieties, Invent. Math., 169(2) : 337 - -400, 2007. 
[GS] Gillet, H. and Soulé, C., Arithmetic analogs of the standard conjectures. Motives (Seattle, WA, 1991), 129 - -140, Proc. Sympos. Pure Math., 55, Part 1, Amer. Math. Soc. Providence, RI, 1994.

[KY1] K. Yamaki, Effective calculation of the geometric height and the Bogomolov conjecture for hyperelliptic curves over function fields, J. Math. Kyoto Univ., 48 - 2: $401--443,2008$.

[KY2] K. Yamaki, Geometric Bogomolov's conjecture for curves of genus 3 over function fields, J. Math. Kyoto Univ., $42-1: 57--81,2002$.

[P] A.N. Paršin, Algebraic curves over function fields, I. Izv. Akad. Nauk SSSR Ser. Mat. 32 : 1191--1219, 1968.

[RB1] R.B. Bapat, Resistance matrix of a weighted graph, MATCH Commun. Math. Comput. Chem. 50 (2004), 73-82.

[RB2] R.B. Bapat, Resistance distance in graphs, The Mathematics Student, 68 (1999), 87-98 pp.

[REU] Summer 2003 Research Experience for Undergraduates (REU) on metrized graphs at the University of Georgia.

[Ru] R. Rumely, Capacity Theory on Algebraic Curves, Lecture Notes in Mathematics 1378, Springer-Verlag, Berlin-Heidelberg-New York, 1989.

[Zh1] S. Zhang, Admissible pairing on a curve, Invent. Math. 112 (1993), 171-193.

[Zh2] S. Zhang, Gross-Schoen cycles and dualising sheaves, preprint, http://www.math.columbia.edu/ szhang/papers/Preprints.htm

(* Mathematica Package Metrized Graph Invariants, i.e, MGI' in short. *)

(*) written by Zubeyir Cinkir *)

(* All functions are Compatible with Mathematica Version 7, and all functions except for TotalLength are compatible with Mathematica Version 6.*)

BeginPackage ["MGI'"]

Valence: :usage = "Valence[A] is a pure function that returns the valence of a given vertex of the metrized graph \[CapitalGamma] with discrete Laplacian A. Therefore, Valence[A] [p] is the valence of the vertex $p$ corresponding to the $\mathrm{p}$-th row of $\mathrm{A}$."

Genus: :usage = "Genus[A] gives the GenusM[A] gives genus of the metrized graph \[CapitalGamma] corresponding to the discerete Laplacian matrix A. This is the same as the 1st Betti number of \[CapitalGamma], which is (number of edges in \[CapitalGamma]) - (number of vertices in \[CapitalGamma]) + 1."

CompleteGraphM: :usage = "CompleteGraphM[n] gives the discrete Laplacian matrix of the complete graph on $\mathrm{n}$ vertices with equal edge lengths such that the total length of the graph is $1 . "$

TotalLength: :usage $=$ "TotalLength[A] gives the total length of the graph \[CapitalGamma] with discrete Laplacian A. The total length of a graph is the sum of its edge lengths."

ResistanceMatrix::usage $=$ "ResistanceM[A] gives the resistance matrix of the graph \[CapitalGamma] with the discrete Laplacian matrix A. ResistanceM[[p,q]] is the effective resistance between the vertices $p$ and $q$, which correspond to the $\mathrm{p}$-th and $\mathrm{q}$-th rows of $\mathrm{A}$. In this case, \[CapitalGamma] is considered as the electric circuit such that the resistances along the edges are given by the edge lengths, and that unit current enters at $p$ and leaves at q."

AdjacentToLaplacian: :usage = "AdjacentToLaplacian[A] AdjacentToLaplacian[A] constructs the discrete Laplacian matrix of the graph with the Adjacency matrix A."

ThetaConstant: : usage $=$ "ThetaConstant $[\mathrm{A}, \mathrm{Q}]$ computes

$\backslash[$ Theta $](\backslash[$ CapitalGamma $])=\backslash ! \backslash(\backslash *$ UnderscriptBox $[\backslash " \backslash[$ Sum $] \backslash "$, RowBox $[\{\backslash " p \backslash ", \backslash ", \backslash ", \backslash " \backslash "$, RowBox $[\{\backslash " q \backslash "$

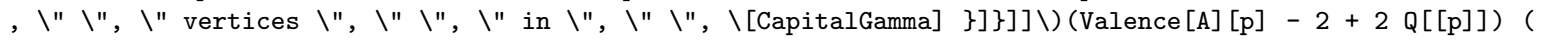
Valence $[A][q]-2+2 Q[q]]) r(p, q)$, where $r(x, y)$ is the resistance function on the graph $\backslash$ [CapitalGamma] with discrete Laplacian $A$. Here $Q$ is either 0 or a list of non-negative integers, in which case the size of $Q$ is equal to the number of rows in A."

TauConstant: :usage = "TauConstant[A] gives the tau constant of the metrized graph \[CapitalGamma] with the discrete Laplacian matrix A." 
PhiConstant: :usage = "PhiConstant [A,Q] computes \[CurlyPhi] ( [CapitalGamma]), where \[CapitalGamma] is the metrized graph with the discrete Laplacian A. Here $Q$ is either 0 or a list of non-negative integers, in which case the size of $Q$ is equal to the number of rows in A."

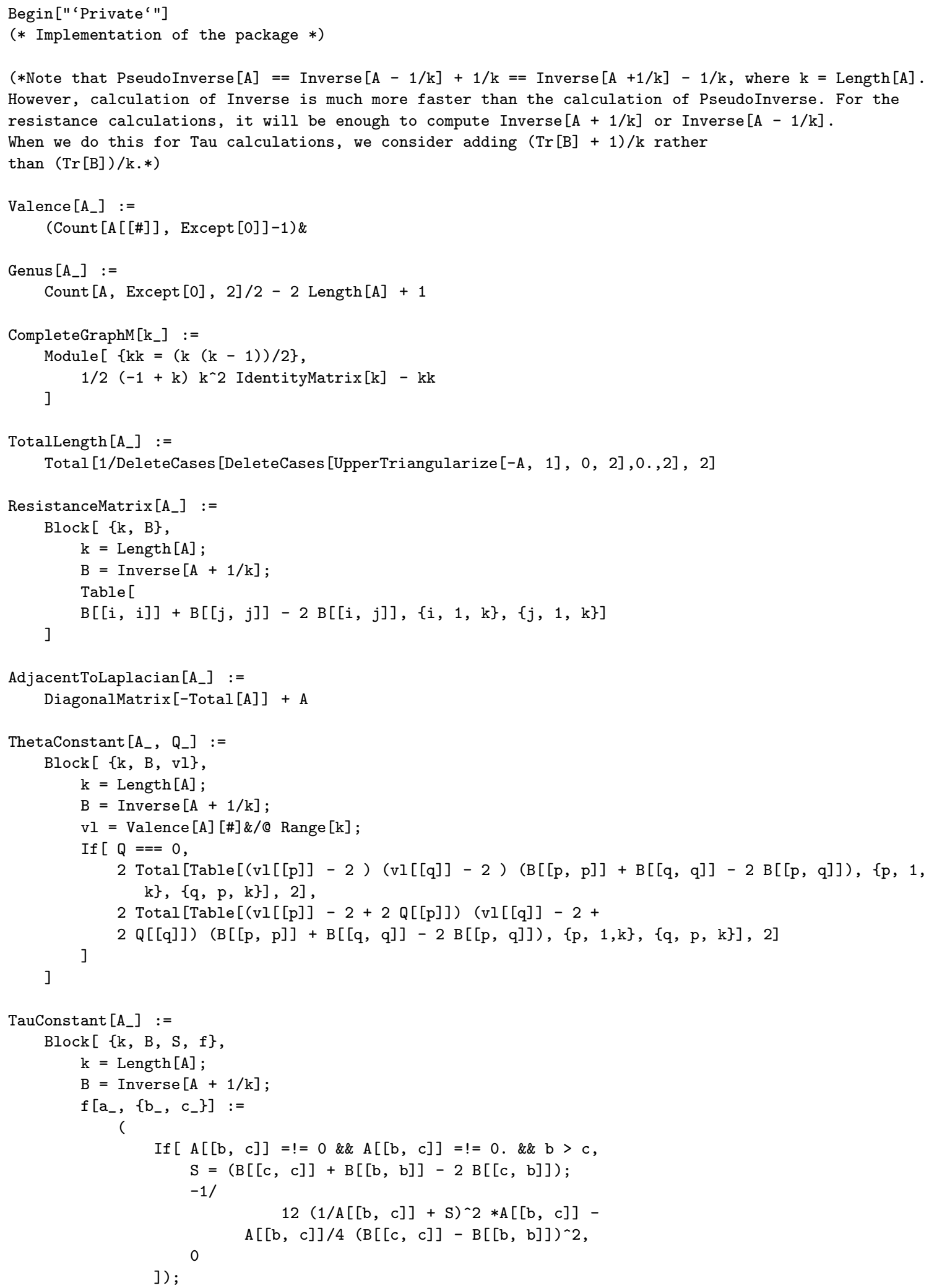


Total [MapIndexed $[f, A,\{2\}], 2]+(\operatorname{Tr}[B]-1) / k$

PhiConstant $\left[\mathrm{A}_{-}, \mathrm{Q}_{-}\right]:=$

Block $[\{k, B, v l, g, f$, theta, tau $\}$,

$\mathrm{k}=$ Length $[\mathrm{A}]$;

$B=$ Inverse $[A+1 / k] ;$

$\mathrm{vl}=$ Valence $[\mathrm{A}][\#] \& / \mathbb{C}$ Range $[\mathrm{k}]$;

$g=\operatorname{If}[Q===0$,

Total $[\mathrm{vl}] / 2-\mathrm{k}+1$,

];

Total $[\mathrm{vl}] / 2-\mathrm{k}+1+\operatorname{Total}[\mathrm{Q}]$

theta $=$

If $[Q===0$,

2 Total $[$ Table $[(\operatorname{vl}[[p]]-2)(v 1[[q]]-2)(B[[p, p]]+$

$B[[q, q]]-2 B[[p, q]]),\{p, 1, k\},\{q, p, k\}], 2]$,

2 Total[Table[(v1[[p]] $-2+2 Q[[p]])(v 1[[q]]-2+$

$2 Q[[q]])(B[[p, p]]+B[[q, q]]-2 B[[p, q]]),\{p, 1$,

];

$\mathrm{k}\},\{\mathrm{q}, \mathrm{p}, \mathrm{k}\}], 2]$

$f\left[a_{-},\left\{b_{-}, c_{-}\right\}\right]:=$

(If $[A[b, c]]=!=0 \& \& A[b, c]]=!=0 . \& \& b>c$,

$S=(B[[c, c]]+B[[b, b]]-2 B[[c, b]]) ;$

$-1 / 12(1 / A[[b, c]]+S) \wedge 2 * A[[b, c]]-$

$A[[b, c]] / 4(B[[c, c]]-B[[b, b]]) \wedge 2$,

]);

tau $=\operatorname{Total}[$ MapIndexed $[f, A,\{2\}], 2]+(\operatorname{Tr}[B]-1) / k$;

]

$(5 \mathrm{~g}-2) / \mathrm{g}$ tau $+\operatorname{theta} /(4 \mathrm{~g})-$ TotalLength $[\mathrm{A}] / 4$

End []

EndPackage []

Zubeyir Cinkir, Wolfram Research, 100 Trade Center, Champaign, Illinois 61820, USA

E-mail address: zubeyirc@wolfram.com 